\title{
Employment of Generalized Receiver for Group-Blind Intersymbol Detection in Downlink CDMA over Fading Multipath Channels
}

\author{
VYACHESLAV TUZLUKOV \\ Department of Technical Maintenance of Aviation and Radio Electronic Equipment \\ Belarusian State Aviation Academy \\ 77, Uborevicha Str., 220096 Minsk \\ BELARUS
}

\begin{abstract}
Group-blind multiuser detectors for uplink code-division multiple-access (CDMA) were developed by Wang and Host-Madsen. These detectors make use of the spreading sequences of known users to construct a group constraint to suppress the intracell interference. However, such techniques demand the estimation of the multipath channels and the delays of the known users. In the present paper, the blind generalized receiver is developed for CDMA in fading multipath channels. The proposed generalized receiver utilizes the correlation information between consecutively received signals to generate the corresponding group constraint. It is shown that by incorporating this group constraint, the proposed generalized receiver can provide different performance gains in both the uplink and downlink environments. Compared with the well-known group-blind detectors, our new methods only need to estimate the multipath channel of the desired user and do not require the channel estimation of other users. Simulation results demonstrate that the proposed generalized receiver outperforms the conventional blind linear multiuser detectors.
\end{abstract}

Key-Words: - Generalized receiver, code-division multiple-access (CDMA), cross-correlation matrix, group constraint, group-blind multiuser detection, intersymbol information, multipath fading.

Received: April 25, 2021. Revised: September 28, 2021. Accepted: October 16, 2021. Published: November 3, 2021.

\section{Introduction}

Multiuser detection for code-division multiple-access (CDMA) systems has been proposed to mitigate multiple access interference (MAI) and enhance channel capacity. The concept of multiuser detection for CDMA systems was first started with the research published in [1], where an optimum multiuser detector for multiple-access Gaussian channels was obtained. However, the optimum detection has never become the main stream because of its unmanageable computational complexity.

Therefore, a number of suboptimum detectors with good performance/complexity trade off were developed during the past decade. In [2] and [3] the adaptive linear decorrelating, or zero-forcing, detector was reported by using training sequences. The linear minimum mean square error (MMSE) detector was another popular suboptimum detector, as presented and analyzed in [4] and [5]. In [6], the basic techniques for multiuser detection were summarized, depicting the evolution of this discipline over the past several years.

Some of the previously mentioned detectors require the information of active users, including the spreading waveform, the multipath channel, and the delay of each user. Unfortunately, such requirements are practically difficult to meet. Hence, the blind multiuser detection, which only requires the information of timing and spreading waveform of the desired user, has received much attention since it was first reported in [7]. Without considering multipath, the blind minimum output energy (MOE) detector was presented in [8]-[10] and then the canonical subspace representation of the decorrelating detector and the MMSE detector were reported in [11]-[13].

In [14], a reduced-rank MOE detector was proposed by using array processing techniques. However, these detectors cannot work properly when the intersymbol interference (ISI) cannot be ignored and the multipath channel is not known. In [15]-[19], several improved subspace approaches were developed based on the channel estimation. A reduced computational constrained optimization solution was proposed in [20]-[25] with a little sacrifice of the performance, i.e., inferior to the performance of MMSE detector.

In [26], several types of group-blind linear detectors were developed to provide substantial performance gains over the blind linear multiuser detection methods for uplink channels. The basic idea behind the group-blind detectors is to suppress the interference from the known users by using their spreading sequences to construct a useful group constraint and to suppress the interference from unknown users ba- 
sed on subspace-based blind methods. Since these detectors need to estimate the multipath channels and the time delays of all known users to obtain the group constraint, computational complexity has to be increased for the implementation of these detectors. In addition, the errors of channel estimation for each known user may deteriorate the performance of these detectors.

The main focus of the present paper is to develop the improved blind detectors for CDMA systems based on the generalized approach to signal processing in noise [27]-[30] in fading multipath channels. It is noted that due to the existence of ISI, especially in asynchronous CDMA systems, the current received signal has indeed certain relationships with its preceeding and succeeding ones. Hence, proper utilization of ISI would be beneficial for blind multiuser detection. The key idea of the proposed detectors is to construct a cross-correlation matrix by exploiting the correlation between the consecutive received signals. By utilizing this cross-correlation matrix, we generate an optimal constraint similar to the group constraint in [26] and then develop the improved blind generalized receiver.

Unlike the detectors in [26], our methods only require the channel estimation of the interested user. Although we mainly focus on the asynchronous CDMA systems in the present paper, it will be shown that for both asynchronous (uplink) and synchronous (downlink) cases, the proposed generalized receiver offers a superior performance to some extent over the conventional blind multiuser detection methods that do not utilize the optimal constraint. The performance improvement of the proposed generalized receiver is justified analytically.

Throughout the present paper, scalars are represented by lower case characters, vectors by bold lower case, and matrices by bold upper case. The following symbols are also used in the present paper:

- $(\cdot)^{T}$ - transpose operator;

- $(\cdot)^{H}$ - Hermitian transpose operator;

- $\operatorname{diag}(\cdot)$ - diagonal matrix;

- $\operatorname{sgn}(\cdot)$ - signum operator;

- $E(\cdot)$ - mathematical expectation.

This paper is organized as follows. Section 2 presents the signal model of asynchronous CDMA systems with multipath. Section 3 discusses the conventional blind multiuser detection methods and their subspace-based implementations. Section 4 presents the generalized receiver and main its functioning condition. Section 5 describes two known blind linear multiuser detectors. In Section 6, simulation examples are provided to demonstrate the performance of the proposed generalized receiver. Section 7 contains the conclusions.

\section{Signal Model}

We consider a DS-CDMA system with $K$ users and a normalized spreading factor of $N$ chips per symbol. The transmitted signal for the $k$-th user is given by

$$
x_{k}(t)=A_{k} \sum_{i=-\infty}^{\infty} b_{k}[i] s_{k}(t-i T)
$$

where $T$ is the symbol duration, and $A_{k}$ and $b_{k}[i] \in[1$, $-1]$ are the amplitude and symbol stream of the $k$-th user, respectively. The spreading waveform $s_{k}(t)$ can be presented in the following form

$$
s_{k}(t)=\sum_{j=0}^{N-1} c_{k}[j] \psi\left(t-j T_{c}\right), \quad 0 \leq t \leq T
$$

where $c_{k}[j]= \pm 1,(0 \leq j \leq N-1)$ is the spreading sequence allocated to the $k$-th user and $\psi(t)$ is the normalized chip waveform with a duration $T_{c}=T / N$. The discrete-time signal for user $k$, generated by matched filtering and sampling at the chip rate, is given by

$$
x_{k}(n)=\sum_{i=-\infty}^{\infty} b_{k}(i) \bar{c}_{k}(n-i N)
$$

where $\bar{c}_{k}(n)=A_{k} c_{k}(n), n=0, \ldots, N-1$. By propagating through the asynchronous multipath channel that is assumed to have the maximum length of $M(M<$ $N$ ) in terms of chip duration, the received discretetime signal $y_{k}(n)$ for user $k$ is given by [31]

$$
y_{k}(n)=\sum_{m=-\infty}^{\infty} x_{k}(m) g_{k}\left(n-d_{k}-m\right)
$$

where $g_{k}(m) \neq 0,0 \leq m \leq M$ is the $m$-th complex channel gain for user $k$ and $0 \leq d_{k}<N$ is the delay of user $k$ in chip periods. Based on (3) and (4), we obtain

$$
\begin{gathered}
y_{k}(n)=\sum_{m=-\infty}^{\infty} b_{k}(m) h_{k}\left(n-d_{k}-m N\right), \\
h_{k}(n)=\sum_{j=-\infty}^{\infty} \bar{c}_{k}(j) g_{k}(n-j),
\end{gathered}
$$

where $h_{k}(n)$ is the signature pulse of user $k$, which is the distorted version of the code $\bar{c}_{k}(n)$ due to the mu- 
ltipath gain $y_{k}(n)$. Finally, the received signal $y_{k}(n)$ is the superposition of the signals from all $K$ users plus the additive complex white Gaussian noise $w(n)$ with the zero mean and variance $\sigma_{w}^{2}$, i.e.

$$
y(n)=\sum_{k=1}^{K} y_{k}(n)+w(n) .
$$

From (6) and denoting

$$
\mathbf{h}_{k, \text { all }}=\left[h_{k}(0), h_{k}(1), \ldots, h_{k}(N-1+M)\right]^{H}
$$

as the signature sequence vector of user $k$, we obtain

$$
\mathbf{h}_{k, \text { all }}=\overline{\mathbf{C}}_{k} \mathbf{g}_{k},
$$

where

$$
\begin{gathered}
\overline{\mathbf{C}}_{k}=\left[\begin{array}{ccc}
\overline{\mathbf{c}}_{k}(0) & \ddots & \mathbf{0} \\
\vdots & \ddots & \overline{\mathbf{c}}_{k}(0) \\
\overline{\mathbf{c}}_{k}(N-1) & \ddots & \vdots \\
\mathbf{0} & \ddots & \overline{\mathbf{c}}_{k}(N-1)
\end{array}\right] ; \\
\mathbf{g}_{k}=\left[\begin{array}{c}
g_{k}(0) \\
\vdots \\
g_{k}(M)
\end{array}\right]
\end{gathered}
$$

In (10), $\overline{\mathbf{C}}_{k}$ is the $(N+M) \times(M+1)$ matrix formed from the product of the spreading sequences and the amplitude of $k$-th user, and $\mathbf{g}_{k}$ is the $k$-th user's multipath channel vector. From (5)-(11), we denote

$$
\begin{gathered}
\mathbf{y}_{k}(n)=\left[\begin{array}{c}
y_{k}(n N) \\
\vdots \\
y_{k}(n N+N-1)
\end{array}\right] ; \\
\mathbf{h}_{k}=\left[\begin{array}{c}
\mathbf{0} \\
h_{k}(0) \\
\vdots \\
h_{k}\left(N-d_{k}-1\right)
\end{array}\right] ; \\
\overline{\mathbf{h}}_{k}=\left[\begin{array}{c}
h_{k}\left(N-d_{k}\right) \\
\vdots \\
h_{k}(N+M-1) \\
\mathbf{0}
\end{array}\right] .
\end{gathered}
$$

It is easy to obtain that

$$
\mathbf{y}_{k}(n)=\mathbf{h}_{k} b_{k}(n)+\overline{\mathbf{h}}_{k} b_{k}(n-1),
$$

where $\mathbf{y}_{k}(n)$ is the $n$-th received signal vector of user $k$. Then, the total received user's signal vector

$$
\mathbf{y}(n)=[y(n N), \ldots, y(n N+N-1)]^{T}
$$

is given by

$$
\begin{gathered}
\mathbf{y}(n)=\sum_{k=1}^{K} \mathbf{y}_{k}(n)+\mathbf{w}(n) \\
=\mathbf{H}_{0} \mathbf{b}(n)+\mathbf{H}_{1} \mathbf{b}(n-1)+\mathbf{w}(n)=\mathbf{H} \overline{\mathbf{b}}(n)+\mathbf{w}(n),
\end{gathered}
$$

where

$$
\left\{\begin{array}{l}
\mathbf{H}_{0}=\left[\mathbf{h}_{1}, \ldots, \mathbf{h}_{K}\right] \\
\mathbf{H}_{1}=\left[\overline{\mathbf{h}}_{1}, \ldots, \overline{\mathbf{h}}_{K}\right] \\
\mathbf{H}=\left[\mathbf{H}_{0} ; \mathbf{H}_{1}\right]
\end{array}\right.
$$

$\mathbf{H}_{0}$ is the signature matrix of the current bits of all users including MAI; $\mathbf{H}_{1}$ is the signature matrix of the previous bits of all users including MAI; $\mathbf{H}$ is the signature matrix of all users;

$$
\left\{\begin{array}{l}
\mathbf{b}(n)=\left[b_{1}(n), \ldots, b_{K}(n)\right]^{T} \\
\mathbf{b}(n-1)=\left[b_{1}(n-1), \ldots, b_{K}(n-1)\right]^{T} \\
\overline{\mathbf{b}}(n)=\left[\mathbf{b}^{T}(n) ; \mathbf{b}^{T}(n-1)\right]^{T}
\end{array}\right.
$$

$\mathbf{b}(n)$ is the vector of current bits of all users; $\mathbf{b}(n-1)$ is the vector of previous bits of all users; $\overline{\mathbf{b}}(n)$ is the vector of bits of all users;

$$
\mathbf{w}(n)=[w(n N), \ldots, w(n N+N-1)]^{T}
$$

is the independent additive white Gaussian noise vector.

In asynchronous (uplink) CDMA wireless communication systems, it is often assumed that $\mathbf{H}_{0}$ and $\mathbf{H}_{1}$ are mutually independent, and $\mathbf{H}$ is the tall matrix with full column rank $2 K$ as the existence of the delays of different users [20], [26]. However, for synchronous (downlink) CDMA wireless communication system, the full-rank condition on $\mathbf{H}$ is hardly satisfied as argued in [32]. It can be explained that in synchronous CDMA wireless communication systems $\left(d_{k}=0\right.$ for $\left.k=1, \ldots, K\right)$, the nonzero elements in the matrix $\mathbf{H}_{1}$ are limited by the number of active users $K$ and the multipath length $M$, i.e., only the $M \times K$ submatrix in the matrix $\mathbf{H}_{1}$ is nonzero. Hence the signature matrix $\mathbf{H}$ will not be full column rank unless $K$ is less than $M$.

\section{Conventional Blind Linear Multiuser Detectors}

The canonical form of a linear detector for user $k$ can be represented by the correlator characterized by 
the vector $\mathbf{v}_{k} \in C^{N}$ followed by the received signal vector $\mathbf{y}(n)$, such that the decision on user $k$ takes the following form

$$
\hat{b}_{k}[n]=\operatorname{sgn}\left\{\mathscr{R}\left(\mathbf{v}_{k}^{H} \mathbf{y}(n)\right)\right\} .
$$

Based on (21), we next introduce the two most popular linear multiuser detectors.

\subsection{Linear decorrelating detector}

The linear decorrelating detector is known as the linear zero-forcing detector. The decorrelating detector is designed to eliminate the MAI and ISI completely at the expense of enhancing the ambient noise. It has the form as in (21) with the weight vector $\mathbf{d}_{k}$ $=\mathbf{v}_{k}$, which is given by

$$
\mathbf{d}_{k}=\underset{\mathbf{d}}{\arg \min }\left\|\mathbf{d}^{H} \mathbf{H}\right\|^{2} \text {, subject to: } \mathbf{d}^{H} \mathbf{h}_{k}=1
$$

where the matrix $\mathbf{H}$ is defined in (17) and $\mathbf{h}_{k}$ is the signature vector of the desired user.

\subsection{Linear MMSE detector}

The linear MMSE detector has the form of (21) with the weight vector $\mathbf{m}_{k}=\mathbf{v}_{k}$, obtained by calculating the minimum output mean squared error, i.e.

$$
\mathbf{m}_{k}=\arg \min _{\mathbf{d}} E\left\{\left|b_{k}[n]-\mathbf{m}^{H} \mathbf{y}[n]\right|^{2}\right\} .
$$

The two detectors can be resolved by subspace methods. Denote the autocorrelation matrix of the received signal in (17) as

$$
\mathbf{Y}=E\left\{\mathbf{y}(n) \mathbf{y}^{H}(n)\right\}=\mathbf{H H}^{H}+\sigma^{2} \mathbf{I}_{N} .
$$

By performing the eigendecomposition of the matrix Y, we obtain

where

$$
\begin{gathered}
\mathbf{Y}=\left[\mathbf{U}_{s} \mathbf{U}_{n}\right]\left|\begin{array}{ll}
\boldsymbol{\Lambda}_{s} & \\
& \boldsymbol{\Lambda}_{n}
\end{array}\right|\left[\mathbf{U}_{s} \mathbf{U}_{n}\right]^{H} \\
=\mathbf{U}_{s} \boldsymbol{\Lambda}_{s} \mathbf{U}_{s}^{H}+\mathbf{U}_{n} \boldsymbol{\Lambda}_{n} \mathbf{U}_{n}^{H},
\end{gathered}
$$

$$
\boldsymbol{\Lambda}_{s}=\operatorname{diag}\left(\lambda_{1}, \ldots, \lambda_{L}\right)
$$

contains the $L$ largest eigenvalues of the matrix $\mathbf{Y}$ and the matrix $\mathbf{U}_{s}$ contains the corresponding orthonormal eigenvectors of the signal subspace spanned by $\mathbf{H H}^{H}$. Both the vectors $\boldsymbol{\Lambda}_{n}=\sigma^{2} \times \mathbf{I}_{N-L}$ and the vectors $\mathbf{U}_{n}$ are the eigenvalues and orthonormal eigenvectors of the noise subspace, respectively. The numerical value of $L$ depends on the column rank of the matrix $\mathbf{H}$ that has the maximum value $2 K$ on the condition that the matrix $\mathbf{H}$ is of full column rank.
By using the eigendecomposition in (25), the detectors in (22) and (23) can be expressed in terms of the subspace forms as described in [11], i.e.

$$
\begin{gathered}
\mathbf{d}_{k}=\mathbf{U}_{s}\left(\boldsymbol{\Lambda}_{s}-\sigma^{2} \mathbf{I}_{L}\right)^{-1} \mathbf{U}_{s}^{H} \mathbf{h}_{k}, \\
\mathbf{m}_{k}=\mathbf{U}_{s} \boldsymbol{\Lambda}_{s}^{-1} \mathbf{U}_{s}^{H} \mathbf{h}_{k} .
\end{gathered}
$$

The signal subspace components $\mathbf{U}_{s}$ and $\boldsymbol{\Lambda}_{s}$ can be estimated from the eigendecomposition of the autocorrelation matrix of the received signal samples. For blind multiuser detection, the detector has only a priori knowledge of the spreading sequence of the desired user. The detectors in (27) and (28) need to estimate $\sigma^{2}$ and $\mathbf{h}_{k}$. By computing the average value of $N-L$ minimum eigenvalues of the matrix $\mathbf{Y}, \sigma^{2}$ can be easily obtained. Meanwhile, when the desired user is synchronized, its signature vector $\mathbf{h}_{k}$ can be estimated in terms of the received signal and the matrix $\overline{\mathbf{C}}_{k}$ in (11), [33]

$$
\begin{gathered}
\mathbf{g}_{k}=\operatorname{Max}-\text { eigenvector }\left(\overline{\mathbf{C}}_{k, u p}^{H} \mathbf{U}_{s} \mathbf{U}_{s}^{H} \overline{\mathbf{C}}_{k, u p}\right), \\
\mathbf{h}_{k}=\overline{\mathbf{C}}_{k, u p} \mathbf{g}_{k},
\end{gathered}
$$

where the matrix $\overline{\mathbf{C}}_{k, u p}$ is composed of the first $N$ rows of the matrix $\overline{\mathbf{C}}_{k}$. Table I summarizes the algorithms of the blind decorrelating detector and blind MMSE detector.

Table I

Signal processing algorithms of subspace blind linear decorrelating and subspace blind linear MMSE detectors

Signal frame: $\mathbf{y}(0), \mathbf{y}(1), \ldots, \mathbf{y}(P)$

STEP 1. Compute autocorrelation

$\mathbf{Y}=\frac{1}{P+1} \sum_{n=0}^{P} \mathbf{y}(n) \mathbf{y}^{H}(n)$

STEP 2. Compute eigendecomposition of the matrix $\mathbf{Y}$

$\mathbf{Y}=\mathbf{U}_{s} \boldsymbol{\Lambda}_{s} \mathbf{U}_{s}^{H}+\sigma^{2} \mathbf{U}_{n} \mathbf{U}_{n}^{H}$

STEP 3. Parameter estimation

$\sigma^{2}=\frac{1}{N-L}($ Sum of themin $N-L$ eigenvalus of the matrix $\mathbf{Y})$

$\mathbf{g}_{k}=\operatorname{Max}$ - eigenvecto $\left(\overline{\mathbf{C}}_{k, u p}^{H} \mathbf{U}_{s} \mathbf{U}_{s}^{H} \overline{\mathbf{C}}_{k, u p}\right)$

$\mathbf{h}_{k}=\overline{\mathbf{C}}_{k, u p} \mathbf{g}_{k}$

STEP 4. Form decorrelating and MMSE detectors

$\mathbf{d}_{k}=\mathbf{U}_{s}\left(\boldsymbol{\Lambda}_{s}-\sigma^{2} \mathbf{I}_{L}\right)^{-1} \mathbf{U}_{s}^{H} \mathbf{h}_{k}$

$\mathbf{m}_{k}=\mathbf{U}_{s} \Lambda_{s}^{-1} \mathbf{U}_{s}^{H} \mathbf{h}_{k}$

\section{Generalized Receiver}

The generalized receiver is constructed in accordance with the generalized approach to signal processing in noise [27]-[30]. The generalized approach to 
signal processing in noise introduces an additional noise source that does not carry any information about the parameters of desired transmitted signal with the purpose to improve the signal processing system performance. This additional noise can be considered as the reference noise without any information about the parameters of the signal to be detected.

The jointly sufficient statistics of the mean and variance of the likelihood function is obtained under the generalized approach to signal processing in noise employment, while the classical and modern signal processing theories can deliver only a sufficient statistics of the mean or variance of the likelihood function. Thus, the generalized approach to signal processing in noise implementation allows us to obtain more information about the parameters of the desired transmitted signal incoming at the generalized receiver input. Owing to this fact, the detectors constructed based on the generalized approach to signal processing in noise technology are able to improve the signal detection performance of signal processing systems in comparison with employment of other conventional detectors.

The generalized receiver (GR) consists of three channels (see Fig.1): the GR correlation detector channel (GR CD) - the preliminary filter (PF), the multipliers 1 and 2, the model signal generator (MSG); the GR energy detector channel (GR ED) the $\mathrm{PF}$, the additional filter (AF), the multipliers 3 and 4 , the summator 1 ; and the GR compensation channel (GR CC) - the summators 2 and 3, the accumulator 1. The threshold apparatus (THRA) device defines the GR threshold.

As we can see from Fig.1, there are two bandpass filters, i.e., the linear systems, at the GR input, namely the PF and AF. We assume for simplicity that these two filters or linear systems have the same amplitude-frequency characteristics or impulse responses. The AF central frequency is detuned relative to the PF central frequency.

There is a need to note the PF bandwidth is matched with the transmitted signal bandwidth. If the detuning value between the PF and AF central frequencies is more than 4 or 5 times the transmitted signal bandwidth to be detected, i.e. $4 \div 5 \Delta f_{s}$, where $\Delta f_{s}$ is the transmitted signal bandwidth, we can believe that the processes at the PF and AF outputs are uncorrelated because the coefficient of correlation between them is negligible (not more than 0.05). This fact was confirmed experimentally in [34] and [35] independently.

Thus, the transmitted signal plus noise can be appeared at the GR PF output and the noise only is appeared at the GR AF output. The stochastic processes at the GR AF and GR PF outputs present the input stochastic samples from two independent frequency-time regions. If the discrete-time noise $w_{i}[k]$ at the GR PF and GR AF inputs is Gaussian, the discrete-time noise $\zeta_{i}[k]$ at the GR PF output is Gaussian too, and the reference discrete-time noise $\eta_{i}[k]$ at the GR AF output is Gaussian owing to the fact that the GR PF and GR AF are the linear systems and we believe that these linear systems do not change the statistical parameters of the input process. Thus, the GR AF can be considered as a generator of the reference noise with a priori information a "no" transmitted signal (the reference noise sample) [28, Chapter 5]. The noise at the GR PF and GR AF outputs can be presented as

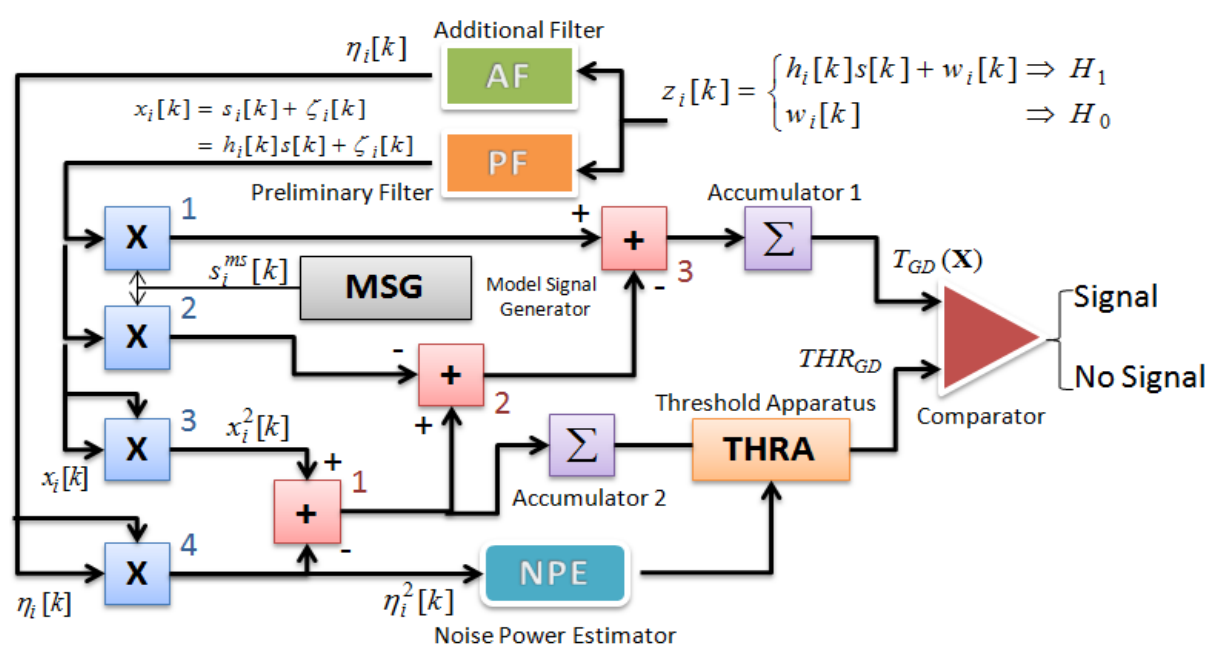

Fig. 1. Generalized receiver. 


$$
\left\{\begin{array}{l}
\zeta_{i}[k]=\sum_{m=-\infty}^{\infty} g_{P F}[m] w_{i}[k-m] ; \\
\eta_{i}[k]=\sum_{m=-\infty}^{\infty} g_{A F}[m] w_{i}[k-m],
\end{array}\right.
$$

where $g_{P F}[m]$ and $g_{A F}[m]$ are the impulse responses of the GR PF and GR AF, respectively.

In a general, under practical implementation of any detector in wireless communication system with sensor array, the bandwidth of the spectrum to be sensed is defined. Thus, the GR AF bandwidth and central frequency can be assigned, too (this bandwidth cannot be used by the transmitted signal because it is out of its spectrum). The case when there are interfering signals within the GR AF bandwidth, the action of this interference on the GR detection performance, and the case of non-ideal condition when the noise at the GR PF and GR AF outputs is not the same by statistical parameters are discussed in [36] and [37].

Under the hypothesis $\mathscr{H}_{1}$ ("a yes" transmitted signal), the GR CD generates the signal component $s_{i}^{m}[k] s_{i}[k]$ caused by interaction between the model signal $s_{i}^{m}[k]$, forming at the MSG output, and the incoming signal $s_{i}[k]$, and the noise component $s_{i}^{m}[k]$ $\times \zeta_{i}[k]$ caused by interaction between the model signal $s_{i}^{m}[k]$ and the noise $\zeta_{i}[k]$ at the PF output. GR ED generates the transmitted signal energy $s_{i}^{2}[k]$ and the random component $s_{i}[k] \zeta_{i}[k]$ caused by interaction between the transmitted signal $s_{i}[k]$ and the noise $\zeta_{i}[k]$ at the PF output. The main purpose of the GR CC is to cancel completely in the statistical sense the GR CD noise component $s_{i}^{m}[k] \zeta_{i}[k]$ and the GR ED random component $s_{i}[k] \zeta_{i}[k]$ based on the same nature of the noise $\zeta_{i}[k]$. The relation between the transmitted signal to be detected $s_{i}[k]$ and the model signal $s_{i}^{m}[k]$ is defined as:

$$
s_{i}^{m}[k]=\mu s_{i}[k],
$$

where $\mu$ is the coefficient of proportionality.

The main functioning condition under the GR employment in any signal processing system including the communication one with radar sensors is the equality between the parameters of the model signal $s_{i}^{m}[k]$ and the incoming signal $s_{i}[k]$, for example, by amplitude. Under this condition it is possible to cancel completely in the statistical sense the noise com- ponent $s_{i}^{m}[k] \zeta_{i}[k]$ of the GR CD and the random component $s_{i}[k] \zeta_{i}[k]$ of the GR ED. Satisfying the GR main functioning condition given by (32), $s_{i}^{m}[k]=$ $s_{i}[k], \mu=1$, we are able to detect the transmitted signal with the high probability of detection at the low $S N R$ and define the transmitted signal parameters with the required high accuracy.

Practical realization of the condition (32) at $\mu \rightarrow$ 1 requires increasing in the complexity of GR structure and, consequently, leads us to increasing in computation cost. For example, there is a need to employ the amplitude tracking system or to use the offline data samples processing. Under the hypothesis $\mathscr{H}_{0}$ ("a no" transmitted signal), satisfying the main GR functioning condition (32) at $\mu \rightarrow 1$ we obtain only the background noise $\eta_{i}^{2}[k]-\zeta_{i}^{2}[k]$ at the GR output.

Under practical implementation, the real structure of GR depends on specificity of signal processing systems and their applications, for example, the radar sensor systems, adaptive wireless communication systems, cognitive radio systems, satellite communication systems, mobile communication systems and so on. In the present paper, the GR circuitry (Fig.1) is demonstrated with the purpose to explain the main functioning principles. Because of this, the GR flowchart presented in the paper should be considered under this viewpoint. Satisfying the GR main functioning condition (32) at $\mu \rightarrow 1$, the ideal case, for the wireless communication systems with radar sensor applications we are able to detect the transmitted signal with very high probability of detection and define accurately its parameters.

In the present paper, we discuss the GR implementation in the broadband space-time spreading MC DS-CDMA wireless communication system. Since the presented GR test statistics is defined by the signal energy and noise power, the equality between the parameters of the model signal $s_{i}^{m}[k]$ and transmitted signal to be detected $s_{i}[k]$, in particular by amplitude, is required that leads us to high circuitry complexity in practice.

For example, there is a need to employ the amplitude tracking system or off-line data sample processing. Detailed discussion about the main GR functioning principles if there is no a priori information and there is an uncertainty about the parameters of transmitted signal, i. e., the transmitted signal parameters are random, can be found in [27], [28, Chapter 6, pp.611-621 and Chapter 7, pp. 631-695]. 
The complete matching between the model signal $s_{i}^{m}[k]$ and the incoming signal $s_{i}[k]$, for example by amplitude, is a very hard problem in practice because the incoming signal $s_{i}[k]$ depends on both the fading and the transmitted signal parameters and it is impractical to estimate the fading gain at the low SNR. This matching is possible in the ideal case only. The GD detection performance will be deteriorated under mismatching in parameters between the model signal $s_{i}^{m}[k]$ and the transmitted signal $s_{i}[k]$ and the impact of this problem is discussed in [38][41], where a complete analysis about the violation of the main GR functioning requirements is presented. The GR decision statistics requires an estimation of the noise variance $\sigma_{\eta}^{2}$ using the reference noise $\eta_{i}[k]$ at the AF output.

Under the hypothesis $\mathscr{H}_{1}$, the signal at the GR PF output, see Fig. 1, can be defined as

$$
x_{i}[k]=s_{i}[k]+\zeta_{i}[k],
$$

where $\zeta_{i}[k]$ is the noise at the PF output and

$$
s_{i}[k]=h_{i}[k] s[k],
$$

where $h_{i}[k]$ are the channel coefficients. Under the hypothesis $\mathscr{H}_{0}$ and for all $i$ and $k$, the process $x_{i}[k]=$ $\zeta_{i}[k]$ at the PF output is subjected to the complex Gaussian distribution and can be considered as the i.i.d. process.

In the ideal case, we can think that the signal at the GR AF output is the reference noise $\eta_{i}[k]$ with the same statistical parameters as the noise $\zeta_{i}[k]$. In practice, there is a difference between the statistical parameters of the noise $\eta_{i}[k]$ and $\zeta_{i}[k]$. How this difference impacts on the GR detection performance is discussed in detail in [28, Chapter 7, pp. 631-695] and in [42], [43],

The decision statistics at the GR output presented in [29] and [30, Chapter 3] is extended for the case of antenna array when an adoption of multiple antennas and antenna arrays is effective to mitigate the negative attenuation and fading effects. The GR decision statistics can be presented in the following form:

$$
\begin{gathered}
T_{G R}(\mathbf{X})=\sum_{k=0}^{N-1} \sum_{i=1}^{M} 2 x_{i}[k] s_{i}^{m}[k] \\
-\sum_{k=0}^{N-1} \sum_{i=1}^{M} x_{i}^{2}[k]+\sum_{k=0}^{N-1} \sum_{i=1}^{M} \eta_{i}^{2}[k] \underset{\substack{\text { F }_{0} \\
\underset{F_{1}}{>}}}{>} T H R_{G R},
\end{gathered}
$$

where

$$
\mathbf{X}=[\mathbf{x}(0), \ldots, \mathbf{x}(N-1)]
$$

is the vector of the random process at the GR PF output and $T H R_{G R}$ is the GR detection threshold.

Under the hypotheses $\mathscr{H}_{1}$ and $\mathscr{H}_{0}$ when the amplitude of the transmitted signal is equal to the amplitude of the model signal, $s_{i}^{m}[k]=s_{i}[k]$ i.e., $\mu=1$, the GR decision statistics $T_{G D}(\mathbf{X})$ takes the following form in the statistical sense, respectively

$$
\left\{\begin{array}{l}
\mathscr{H}_{1}: T_{G D}(\mathbf{X})=\sum_{k=0}^{N-1} \sum_{i=1}^{M}\left\{s_{i}^{2}[k]+\eta_{i}^{2}[k]-\zeta_{i}^{2}[k]\right\} ; \\
\mathscr{H}_{0}: T_{G D}(\mathbf{X})=\sum_{k=0}^{N-1} \sum_{i=1}^{M}\left\{\eta_{i}^{2}[k]-\zeta_{i}^{2}[k]\right\} .
\end{array}\right.
$$

In (37) the term $\sum_{k=0}^{N-1} \sum_{i=1}^{M} s_{i}^{2}[k]=E_{s}$ corresponds to the average transmitted signal energy, and the term $\sum_{k=0}^{N-1} \sum_{i=1}^{M} \eta_{i}^{2}[k]-\sum_{k=0}^{N-1} \sum_{i=1}^{M} \zeta_{i}^{2}[k]$ is the background noise at the GR output. The GR output background noise is a difference between the noise power at the GR PF and GR AF outputs. Practical implementation of the GR decision statistics requires an estimation of the noise variance $\sigma_{\eta}^{2}$ using the reference noise $\eta_{i}[k]$ at the AF output.

\section{Blind Generalized Receiver}

The conventional generalized receiver discussed in Section 4 only knows the spreading sequence of the desired user. However, some performance gains may be obtained if the generalized detector has the knowledge of the spreading sequences of a group of users. Such an expectation has been achieved in [26], where several group-blind linear multiuser detectors were presented. These detectors first exploit the known user spreading sequences to estimate their signature vectors based on the methods in (29) and (30). By using the estimated signal vectors, several group-blind generalized receivers can be developed with the following group constraint:

$$
\mathbf{T}_{k}^{H} \widetilde{\mathbf{H}}=\mathbf{1}_{P, k}^{T},
$$

where $P$ is the number of columns in the matrix $\widetilde{\mathbf{H}}$, $\mathbf{T}_{k}$ is the vector of decision statistics of the generalized receiver defined by (35) for user $k$, the matrix $\widetilde{\mathbf{H}}$ contains the estimated signature vectors of a group of known users, which is the subset of the matrix $\mathbf{H}$ in (17), and $\mathbf{1}_{P, k}$ is denoted as the $P$ dimension column vector with all zero entries except for the $k$-th 
entry. The constraint in (38) is the key idea of the group-blind zero-forcing generalized receiver and the group-blind hybrid detector in [26]. Equation (38) is equivalent to the following constraints:

$$
\left\{\begin{array}{l}
\mathbf{T}_{k}^{H} \mathbf{h}_{k}=1 ; \\
\mathbf{T}_{k}^{H} \hat{\mathbf{H}}=0,
\end{array}\right.
$$

where the matrix $\hat{\mathbf{H}}$ contains the remaining column vectors of the matrix $\widetilde{\mathbf{H}}$ except for the vector $\mathbf{h}_{k}$. In (39), the first constraint is to assure the existence of the desired user signal at the generalized receiver output, and the second constraint is used to zero force the interference of the known user's signal. Hence, the essence of the group-blind multiuser detection is to construct a group constraint, which exactly removes the influence of some interfering users, thereby achieving performance gains. Since the group constraint in [26] requires the known user's spreading sequences and the estimations of their multipath channels and delays, such techniques are only fit for the CDMA up-link, where the base station knows the spreading sequences of the intracell users.

Inspired from the previously mentioned group methods, we develop two improved blind generalized receivers in this section. The basic idea for the proposed generalized receivers is to construct a new group constraint, which is similar to the group constraint in (39), by exploiting the intersymbol information of the received signals. In this method, we only need the channel estimation of the desired user. Therefore, our generalized receivers can provide performance gain over the blind linear decorrelating detector and the blind linear MMSE detector for both the downlink and uplink cases.

Let us assume without loss of generality that user 1 is the desired user, and the generalized receiver is synchronized to the user 1 , i.e., $d_{1}=0$. Then the signal in (17) can be rewritten in the following form:

$$
\mathbf{y}(n)=\mathbf{h}_{1} b_{1}(n)+\overline{\mathbf{h}}_{1} b_{1}(n-1)+\breve{\mathbf{H}} \breve{\mathbf{b}}(n)+\mathbf{w}(n),
$$

where

$$
\breve{\mathbf{H}}=\left[\mathbf{h}_{2}, \ldots, \mathbf{h}_{K}, \overline{\mathbf{h}}_{2}, \ldots, \overline{\mathbf{h}}_{K}\right],
$$

is the signature matrix and

$$
\breve{\mathbf{b}}(n)=\left[b_{2}(n), \ldots, b_{K}(n), b_{2}(n-1), \ldots, b_{K}(n-1)\right]^{T}
$$

is the vector of received signals of the interfering users. We next consider the channel estimation of $\mathbf{h}_{1}$, which is the signature vector of the desired signal $b_{1}(n)$. Meanwhile, we will also estimate the vector $\overline{\mathbf{h}}_{1}$ since it is required by the following derived dete- ctors. With the assumption that the user 1 is synchronized, we denote

$$
\begin{aligned}
\overline{\mathbf{C}}_{1, u p} & =\left[\begin{array}{cccc}
\overline{\mathbf{c}}_{1}(0) & \cdots & \mathbf{0} \\
\vdots & \ddots & \overline{\mathbf{c}}_{1}(0) \\
\vdots & \ddots & \vdots \\
\overline{\mathbf{c}}_{1}(N-1) & \cdots & \overline{\mathbf{c}}_{1}(N-M-1)
\end{array}\right], \\
\overline{\mathbf{C}}_{1, \text { down }} & =\left[\begin{array}{cccc}
0 & \overline{\mathbf{c}}_{1}(N-1) & \cdots & \overline{\mathbf{c}}_{1}(N-M) \\
\vdots & \vdots & \ddots & \\
\vdots & \mathbf{0} & \cdots & \overline{\mathbf{c}}_{1}(N-1) \\
0 & \cdots & \cdots & 0
\end{array}\right],
\end{aligned}
$$

where both $\overline{\mathbf{C}}_{1, \text { up }}$ and $\overline{\mathbf{C}}_{1, \text { down }}$ are the $N \times(M+1)$ matrices. The matrix $\overline{\mathbf{C}}_{1, u p}$ is constructed by the first $N$ rows of the matrix $\overline{\mathbf{C}}_{1}$ defined in (11), and the matrix $\overline{\mathbf{C}}_{1, \text { down }}$ is formed by the remaining $M$ rows of the matrix $\overline{\mathbf{C}}_{1}$ plus the $(N-M) \times(M+1)$ zero matrix.

According to the definitions of the vetors $\mathbf{h}_{1}$ and $\overline{\mathbf{h}}_{1}$ in Section 2 and the denotation in (43) and (44), it is easy to obtain that

$$
\begin{aligned}
& \mathbf{h}_{1}=\overline{\mathbf{C}}_{1, u p} \mathbf{g}_{1}, \\
& \overline{\mathbf{h}}_{1}=\overline{\mathbf{C}}_{1, \text { down }} \mathbf{g}_{1},
\end{aligned}
$$

where the vector $\mathbf{g}_{1}$ is the multipath channel of user 1 , which is defined in (11). Since the matrices $\overline{\mathbf{C}}_{1, u p}$ and $\overline{\mathbf{C}}_{1, \text { down }}$ are easy to construct with the a priori knowledge of the spreading sequences of the desired user, we only need to consider the estimation of the vector $\mathbf{g}_{1}$. The multipath channel vector $\mathbf{g}_{1}$ can be estimated by exploiting the orthogonality between the signal subspace and the noise subspace. Since the vector $\mathbf{h}_{1}$ is the subspace of the matrix $\mathbf{U}_{s}$ in (25), and the subspace of the matrix $\mathbf{U}_{s}$ is orthogonal to the matrix $\mathbf{U}_{n}$, we have

$$
\mathbf{U}_{n}^{H} \mathbf{h}_{1}=\mathbf{U}_{n}^{H} \overline{\mathbf{C}}_{1, u p} \mathbf{g}_{1}=\mathbf{0} .
$$

Hence, the estimation of the vector $\mathbf{g}_{1}$ can be uniquely determined by computing the principle eigenvector of the matrix $\overline{\mathbf{C}}_{1, u p}^{H} \mathbf{U}_{s} \mathbf{U}_{s}^{H} \overline{\mathbf{C}}_{1, u p}$ [12], [28], i.e.

$$
\hat{\mathbf{g}}_{1}=\operatorname{Max} \text { - eigenvector }\left(\overline{\mathbf{C}}_{1, u p}^{H} \mathbf{U}_{s} \mathbf{U}_{s}^{H} \overline{\mathbf{C}}_{1, \text { up }}\right) .
$$

Based on (48), the signature vectors $\mathbf{h}_{1}$ and $\overline{\mathbf{h}}_{1}$ can be determined according to (45) and (46).

After the channel estimation, we construct the useful cross-correlation matrix $\overline{\mathbf{Y}}$ given by

$$
\overline{\mathbf{Y}}=E\left\{\mathbf{y}(n) \mathbf{y}(n+1)^{H}\right\}+\mathrm{E}\left\{\mathbf{y}(n) \mathbf{y}(n-1)^{H}\right\}^{H}
$$




$$
=\mathbf{H}\left[\begin{array}{cc}
\mathbf{0} & \mathbf{I}_{K} \\
\mathbf{I}_{K} & \mathbf{0}
\end{array}\right] \mathbf{H},
$$

where the matrix $\overline{\mathbf{Y}}$ is generated by the cross-correlation of the received signal. It is seen that the matrix $\overline{\mathbf{Y}}$ is the $N \times N$ matrix and is irrelevant to the background noise of the generalized receiver. This matrix plays an important role in the present paper since it will be exploited to develop the proposed generalized receivers.

Recalling that the basic idea of the group-blind multiuser detection is to suppress the interference of some known users based on the group constraint, we, naturally, expect that the more subspace of the interfering users the constraint removes, the better performance improvement the detector achieves. In this section, we utilize the matrix $\overline{\mathbf{Y}}$ to develop a constraint similar to the group constraint in (38) for the desired user, which is given by

$$
\mathbf{T}_{1}^{H} \overline{\mathbf{Y}}=\overline{\mathbf{h}}_{1}^{H} .
$$

Let us now consider the properties of the above constraint with the following propositions to demonstrate analytically the performance improvement brought by the proposed constraint.

Proposition 1: Based on the signal model given by (17) and the definition of the matrix $\overline{\mathbf{Y}}$ in (49), the constraint $\mathbf{T}_{1}^{H} \mathbf{H}=\mathbf{1}_{2 K, 1}^{T}$ implies the constraint $\mathbf{T}_{1}^{H} \overline{\mathbf{Y}}=$ $\overline{\mathbf{h}}_{1}^{H}$, i.e.

$$
\mathbf{T}_{1}^{H} \mathbf{H}=\mathbf{1}_{2 K, 1}^{T} \Rightarrow \mathbf{T}_{1}^{H} \overline{\mathbf{Y}}=\overline{\mathbf{h}}_{1}^{H} .
$$

Proof: The proof is straightforward by directly substituting the constraint $\mathbf{T}_{1}^{H} \mathbf{H}=\mathbf{1}_{2 K, 1}^{T}$ into the constraint $\mathbf{T}_{1}^{H} \overline{\mathbf{Y}}$, i.e.

$$
\begin{gathered}
\mathbf{T}_{1}^{H} \overline{\mathbf{Y}}=\mathbf{T}_{1}^{H} \mathbf{H}\left\lfloor\begin{array}{cc}
\mathbf{0} & \mathbf{I}_{K} \\
\mathbf{I}_{K} & \mathbf{0}
\end{array}\right\rfloor \mathbf{H}^{H}=\mathbf{1}_{2 K, 1}^{T}\left\lfloor\begin{array}{cc}
\mathbf{0} & \mathbf{I}_{K} \\
\mathbf{I}_{K} & \mathbf{0}
\end{array}\right\rfloor \mathbf{H}^{H} ; \\
\mathbf{1}_{2 K, K+1}^{T} \mathbf{H}^{H}=\overline{\mathbf{h}}_{1}^{H},
\end{gathered}
$$

where the last equality follows from the definition of the matrix $\mathbf{H}$ in (17), i.e.

$$
\mathbf{H}[:, K+1]=\overline{\mathbf{h}}_{1} .
$$

The constraint $\mathbf{T}_{1}^{H} \mathbf{H}=\mathbf{1}_{2 K, 1}^{T}$ is the optimal group constraint for group-blind multiuser detection since it ensures that the decision statistics of the generalized receiver $\mathbf{T}_{1}$ is orthogonal to the subspace spanned by all interfering users except the desired user. The result of the Proposition 1 shows that the proposed constraint is the necessary condition of the optimal group constraint. It means that the proposed constra- $\operatorname{int} \mathbf{T}_{1}^{H} \overline{\mathbf{Y}}=\overline{\mathbf{h}}_{1}^{H}$ will not remove the subspace spanned by the desired user and it is possible to achieve the optimal group constraint based on this constraint. Therefore, we are interested in the feature of the proposed constraint and want to know if this constraint implies the optimal group constraint or to what extent it removes the subspace spanned by the interfering users. Propositions 2-4 summarize the proposed constraint's ability to resist the interference brought by the interfering users in different cases.

Proposition 2: The constraint $\mathbf{T}_{1}^{H} \overline{\mathbf{Y}}=\overline{\mathbf{h}}_{1}^{H}$ implies the constraint $\mathbf{T}_{1}^{H} \mathbf{H}=\mathbf{1}_{2 K, 1}^{T}$ if the matrix $\mathbf{H}$ has full column rank $2 K$.

Proof: We proceed with a proof by contradiction. Assuming that $\mathbf{T}_{1}^{H} \overline{\mathbf{Y}}=\overline{\mathbf{h}}_{1}^{H}$ and $\mathbf{T}_{1}^{H} \mathbf{H} \neq \mathbf{1}_{2 K, 1}^{T}$, then the constraint $\mathbf{T}_{1}^{H} \mathbf{H}$ can be expressed in the following form

$$
\mathbf{T}_{1}^{H} \mathbf{H}=\mathbf{1}_{2 K, 1}^{T}+\mathbf{f}^{H},
$$

where

$$
\mathbf{f}=\left[\mathbf{f}_{u p}^{T}, \mathbf{f}_{d o w n}^{T}\right]^{T}
$$

is a nonzero $2 K$ dimensional column vector, and the vectors $\mathbf{f}_{u p}$ and $\mathbf{f}_{\text {down }}$ are the first $K$ entries and the last $K$ entries of the vector $\mathbf{f}$, respectively. By denoting

$$
\breve{\mathbf{f}}=\left[\mathbf{f}_{d o w n}^{T}, \mathbf{f}_{u p}^{T}\right]
$$

and substituting (54) into the constraint $\mathbf{T}_{1}^{H} \overline{\mathbf{Y}}$, we obtain

$$
\begin{gathered}
\mathbf{T}_{1}^{H} \overline{\mathbf{Y}}=\overline{\mathbf{h}}_{1}^{H} \Rightarrow \mathbf{T}_{1}^{H} \mathbf{H}\left[\begin{array}{cc}
\mathbf{0} & \mathbf{I}_{K} \\
\mathbf{I}_{K} & \mathbf{0}
\end{array}\right] \mathbf{H}^{H}=\overline{\mathbf{h}}_{1}^{H} \\
\Rightarrow\left(\mathbf{1}_{2 K, 1}^{T}+\mathbf{f}^{H}\right)\left[\begin{array}{cc}
\mathbf{0} & \mathbf{I}_{K} \\
\mathbf{I}_{K} & \mathbf{0}
\end{array}\right] \mathbf{H}^{H}=\overline{\mathbf{h}}_{1}^{H} \\
\Rightarrow\left(\mathbf{1}_{2 K, K+1}^{T}+\breve{\mathbf{f}}^{H}\right) \mathbf{H}^{H}=\overline{\mathbf{h}}_{1}^{H} \Rightarrow \overline{\mathbf{h}}_{1}^{H}+\breve{\mathbf{f}}^{H} \mathbf{H}^{H}=\overline{\mathbf{h}}_{1}^{H} \\
\Rightarrow \breve{\mathbf{f}}=0 \Rightarrow \mathbf{f}=0,
\end{gathered}
$$

where the third right arrow follows from the fact that

$$
\mathbf{f}^{H}\left[\begin{array}{cc}
\mathbf{0} & \mathbf{I}_{K} \\
\mathbf{I}_{K} & \mathbf{0}
\end{array}\right]=\breve{\mathbf{f}}^{H}
$$

and the fifth right arrow is based on the fact that the matrix $\mathbf{H}$ is of full column rank. Hence, Eq.(57) contradicts Eq. (54).

Proposition 2 shows that the proposed constraint is equivalent to the optimal group constraint which removes all MAI and ISI on the condition that the matrix $\mathbf{H}$ is of full column rank. Recalling that the 
signature matrix $\mathbf{H}$ in the asynchronous (uplink) CDMA wireless communication system model, which is given in Section 2, is assumed to be full rank, we naturally expect that the proposed constraint provides the greatest performance gain on the uplink of CDMA wireless communication system.

As mentioned before, the full-rank condition on the matrix $\mathbf{H}$ is hardly satisfied in the downlink of CDMA wireless communication system, where the intracell users are transmitted synchronously and the intercell users are transmitted asynchronously. In this case, we divide the matrix $\mathbf{H}$ into the matrices $\mathbf{H}_{0}$ and $\mathbf{H}_{1}$ according to (17), where the matrix $\mathbf{H}_{0}$ contains the subspace spanned by the desired user and MAI, and the matrix $\mathbf{H}_{1}$ contains the subspace spanned by ISI. In the downlink of CDMA wireless communication system, most treatments assume that the matrix $\mathbf{H}_{0}$ is the full rank matrix and independent of the matrix $\mathbf{H}_{1}$, while the matrix $\mathbf{H}_{1}$ is not full rank matrix when the number of active users $K$ exceeds the maximum length of the multipath channel $M$.

Proposition 3: For the nonfull-rank matrix $\mathbf{H}$, the constraint $\mathbf{T}_{1}^{H} \overline{\mathbf{Y}}=\overline{\mathbf{h}}_{1}^{H}$ implies $\mathbf{T}_{1}^{H} \mathbf{H}_{1}=\mathbf{0}$ if the matrix $\mathbf{H}_{0}$ is independent of the matrix $\mathbf{H}_{1}$ with full column rank.

Proof: The proof is similar to the Proof of Proposition 2, where

$$
\begin{gathered}
\mathbf{T}_{1}^{H} \overline{\mathbf{Y}}=\overline{\mathbf{h}}_{1}^{H} \Rightarrow \mathbf{T}_{1}^{H} \mathbf{H}\left[\begin{array}{rr}
\mathbf{0} & \mathbf{I}_{K} \\
\mathbf{I}_{K} & \mathbf{0}
\end{array}\right] \mathbf{H}^{H}=\overline{\mathbf{h}}_{1}^{H} \\
\Rightarrow \mathbf{T}_{1}^{H}\left[\begin{array}{ll}
\mathbf{H}_{0} & \mathbf{H}_{1}
\end{array}\right]\left[\begin{array}{cc}
\mathbf{0} & \mathbf{I}_{K} \\
\mathbf{I}_{K} & \mathbf{0}
\end{array}\right]\left[\begin{array}{ll}
\mathbf{H}_{0} & \mathbf{H}_{1}
\end{array}\right]^{H}=\overline{\mathbf{h}}_{1}^{H} \\
\Rightarrow \mathbf{T}_{1}^{H} \mathbf{H}_{0} \mathbf{H}_{1}^{H}+\mathbf{T}_{1}^{H} \mathbf{H}_{1} \mathbf{H}_{0}^{H}=\overline{\mathbf{h}}_{1}^{H} \Rightarrow \mathbf{T}_{1}^{H} \mathbf{H}_{1} \mathbf{H}_{0}^{H}=\mathbf{0} \\
\Rightarrow \mathbf{T}_{1}^{H} \mathbf{H}_{1}=0,
\end{gathered}
$$

where the fourth arrow follows from the fact that the vector $\overline{\mathbf{h}}_{1} \in$ the range $\left(\mathbf{H}_{1}\right)$ and the matrix $\mathbf{H}_{0}$ is independent of the matrix $\mathbf{H}_{1}$, and the last arrow is based on the fact that the matrix $\mathbf{H}_{0}$ is of full column rank.

Proposition 3 shows that the proposed constraint can totally remove the ISI with the nonfull rank matrix $\mathbf{H}$ in the downlink of CDMA wireless communication system. In particular, if we assumed that the vector $\overline{\mathbf{h}}_{1}$ is independent of other vectors in the matrix $\mathbf{H}_{1}$, a more satisfying result can be achieved by the proposed constraint.

Proposition 4: For the nonfull-rank matrix $\mathbf{H}$, the constraint $\mathbf{T}_{1}^{H} \overline{\mathbf{Y}}=\overline{\mathbf{h}}_{1}^{H}$ implies $\mathbf{T}_{1}^{H} \mathbf{H}_{1}=\mathbf{1}_{2 K, 1}^{T}$ if the matrix $\mathbf{H}_{0}$ is independent of the matrix $\mathbf{H}_{1}$ and the vector $\overline{\mathbf{h}}_{1}$ is independent of the rest vectors in the matrix $\mathbf{H}_{1}$.

Proof: The proof is similar to the Proof of Proposition 3, i.e., we consider

$$
\begin{gathered}
\mathbf{T}_{1}^{H} \overline{\mathbf{Y}}=\overline{\mathbf{h}}_{1}^{H} \\
\Rightarrow \mathbf{T}_{1}^{H}\left[\begin{array}{ll}
\mathbf{H}_{0} & \mathbf{H}_{1}
\end{array}\right]\left[\begin{array}{cc}
\mathbf{0} & \mathbf{I}_{K} \\
\mathbf{I}_{K} & \mathbf{0}
\end{array}\right]\left[\begin{array}{ll}
\mathbf{H}_{0} & \mathbf{H}_{1}
\end{array}\right]^{H}=\overline{\mathbf{h}}_{1}^{H} \\
\Rightarrow \mathbf{T}_{1}^{H} \mathbf{H}_{0} \mathbf{H}_{1}^{H}+\mathbf{T}_{1}^{H} \mathbf{H}_{1} \mathbf{H}_{0}^{H}=\overline{\mathbf{h}}_{1}^{H} \Rightarrow \mathbf{T}_{1}^{H} \mathbf{H}_{1} \mathbf{H}_{0}^{H}=\overline{\mathbf{h}}_{1}^{H} \\
\Rightarrow \mathbf{T}_{1}^{H} \mathbf{H}_{1}=\mathbf{1}_{2 K, 1}^{T},
\end{gathered}
$$

where the third arrow is derived from the independence between the matrices $\mathbf{H}_{0}$ and $\mathbf{H}_{1}$, and the last arrow is based on the fact that the vector $\overline{\mathbf{h}}_{1}$ cannot be constructed by the linear combination of other vectors in the matrix $\mathbf{H}_{1}$.

The Proposition 4 shows that the proposed constraint can remove all MAI in the statistical; sense if the signature vector of the desired user in the ISI matrix $\mathbf{H}_{1}$ is independent of the other signature vectors in ISI. In this case, we do not require the full rank of the matrix $\mathbf{H}_{0}$. However, if we also assumed that the matrix $\mathbf{H}_{0}$ is of full rank, the constraint in the Proposition 4 can eliminate both MAI and ISI according to the Proposition 3, which means that our proposed constraint may achieve the optimal group constraint on certain conditions even without the full rank matrix $\mathbf{H}$.

The results discussed in the Propositions 2-4 show that the proposed constraint $\mathbf{T}_{1}^{H} \overline{\mathbf{Y}}=\overline{\mathbf{h}}_{1}^{H}$ is able to remove the MAI and ISI in the statistical sense to different extents according to the properties of the signature matrix $\mathbf{H}$. In this case, we develop two improved blind generalized detectors by considering this constraint.

\subsection{Improved blind generalized receiver with decorrelating}

The improved blind generalized receiver with décorrelation considers the minimization of the following constraint cost function in the statistical sense

$$
\begin{gathered}
\mathbf{T}_{\text {opt }}^{d}=\underset{\mathbf{T}}{\operatorname{argmin}}\left|\mathbf{T}^{H} \mathbf{H}\right|^{2}, \quad \mathbf{T} \in \operatorname{range}(\mathbf{H}) \\
\text { subject to: }\left\{\begin{array}{l}
\mathbf{T}^{H} \mathbf{h}_{1}=\mathbf{1}, \\
\mathbf{T}^{H} \overline{\mathbf{Y}}=\overline{\mathbf{h}}_{1}^{H},
\end{array}\right.
\end{gathered}
$$

where $\mathbf{T}_{o p t}^{d}$ is the decision statistics matrix of the proposed generalized receiver. The first constraint in (61) is used to keep the information of the desired 
user, and the second constraint is used to suppress the MAI and ISI in the statistical sense. The two constraints compose an equivalent group-blind constraint to (39). As it was shown in [38] and [39] the improved blind generalized receiver with decorrelating in (61) is equivalent to the decorrelating detector in (22) by physical sense, i.e. $\mathbf{T}_{o p t}^{d} \approx \mathbf{d}_{1}$. The Proposition 5 presents the resolution of the proposed blind generalized receiver with the decorrelating.

Proposition 5: Based on the eigendecomposition in (25), the solution of the improved blind generalized receiver with decorrelating in (61) is given by

$$
\mathbf{T}_{o p t}^{d}=\mathbf{U}_{s}\left(\boldsymbol{\Lambda}_{s}-\sigma^{2} \mathbf{I}_{L}\right)^{-1} \mathbf{U}_{s}^{H}\left(\boldsymbol{\lambda}_{1} \mathbf{h}_{1}+\boldsymbol{\lambda}_{2} \overline{\mathbf{Y}}\right),
$$

where

$$
\left\{\begin{array}{l}
\boldsymbol{\lambda}_{2}=\left[\boldsymbol{\alpha} \overline{\mathbf{Y}}^{H} \mathbf{U}_{s}\left(\boldsymbol{\Lambda}_{s}-\sigma^{2} \mathbf{I}_{L}\right)^{-1} \mathbf{U}_{s}^{H} \overline{\mathbf{Y}}-\boldsymbol{\theta} \boldsymbol{\theta}^{H}\right]^{\bar{*}}\left[\boldsymbol{\alpha} \overline{\mathbf{h}}_{1}-\boldsymbol{\theta}\right] \\
\boldsymbol{\lambda}_{1}=\boldsymbol{\alpha}^{-1}\left[1-\boldsymbol{\theta}^{H} \boldsymbol{\lambda}_{2}\right] \\
\boldsymbol{\theta}=\overline{\mathbf{Y}}^{H} \mathbf{U}_{s}\left(\boldsymbol{\Lambda}_{s}-\sigma^{2} \mathbf{I}_{L}\right)^{-1} \mathbf{U}_{s}^{H} \mathbf{h}_{1} ; \\
\boldsymbol{\alpha}=\mathbf{h}_{1}^{H} \mathbf{U}_{s}\left(\boldsymbol{\Lambda}_{s}-\sigma^{2} \mathbf{I}_{L}\right)^{-1} \mathbf{U}_{s}^{H} \mathbf{h}_{1}
\end{array}\right.
$$

The symbol $(\bar{*})$ denotes the pseudo-inverse.

Proof: See the Appendix 1.

\subsection{Improved blind hybrid generalized receiver}

The improved blind hybrid generalized receiver considers the following constrained optimization problem:

$$
\begin{gathered}
\mathbf{T}_{\text {opt }}^{m}=\underset{\mathbf{m}}{\arg \min } E\left\{\left|b_{1}(n)-\mathbf{T}^{H} \mathbf{y}_{1}(n)\right|^{2}\right\}, \\
\mathbf{T} \in \operatorname{range}(\mathbf{H}) \text { subject to }\left\{\begin{array}{l}
\mathbf{T}^{H} \mathbf{h}_{1}=\mathbf{1} ; \\
\mathbf{T}^{H} \overline{\mathbf{Y}}=\overline{\mathbf{h}}_{1}^{H},
\end{array}\right.
\end{gathered}
$$

where matrix $\mathbf{T}_{o p t}^{m}$ is the decision statistics of the proposed blind hybrid generalized receiver. The Proposition 6 gives an implementation for the proposed blind hybrid generalized receiver.

$$
\mathbf{T}_{\text {opt }}^{m}=\mathbf{U}_{s}\left(\boldsymbol{\Lambda}_{s}^{-1} \mathbf{U}_{s}^{H}\left(\overline{\boldsymbol{\lambda}}_{1} \mathbf{h}_{1}+\overline{\boldsymbol{\lambda}}_{2} \overline{\mathbf{Y}}\right),\right.
$$

where

$$
\left\{\begin{array}{l}
\overline{\boldsymbol{\lambda}}_{2}=\left[\overline{\boldsymbol{\alpha}} \overline{\mathbf{Y}}^{H} \mathbf{U}_{s}\left(\boldsymbol{\Lambda}_{s}^{-1} \mathbf{U}_{s}^{H} \overline{\mathbf{Y}}-\overline{\boldsymbol{\theta}} \overline{\boldsymbol{\theta}}^{H}\right]^{*}\left[\overline{\boldsymbol{\alpha}} \overline{\mathbf{h}}_{1}-\overline{\boldsymbol{\theta}}\right] ;\right. \\
\overline{\boldsymbol{\lambda}}_{1}=\overline{\boldsymbol{\alpha}}^{-1}\left[1-\overline{\boldsymbol{\theta}}^{H} \boldsymbol{\lambda}_{2}\right] ; \\
\overline{\boldsymbol{\theta}}=\overline{\mathbf{Y}}^{H} \mathbf{U}_{s}\left(\boldsymbol{\Lambda}_{s}-\sigma^{2} \mathbf{I}_{L}\right)^{-1} \mathbf{U}_{s}^{H} \mathbf{h}_{1} ; \\
\overline{\boldsymbol{\alpha}}=\mathbf{h}_{1}^{H} \mathbf{U}_{s}\left(\boldsymbol{\Lambda}_{s}-\sigma^{2} \mathbf{I}_{L}\right)^{-1} \mathbf{U}_{s}^{H} \mathbf{h}_{1} .
\end{array}\right.
$$

Proof: See the Appendix 2.
In the above-mentioned results, two improved blind generalized receivers are expressed in subspace forms. In these detectors, the influence of interfering users is mitigated by the two constraints, which are constructed from the correlation matrix $\overline{\mathbf{Y}}$ and the channel estimation of the desired user. After suppressing the interfering signals, the signals of the desired user are identified by the transformation of the subspace based on the zero-forcing or the hybrid criteria. Unlike the group constraint in [26], our proposed constraints utilize the correlation matrix $\overline{\mathbf{Y}}$ to improve the performance of the detectors and then avoid the channel estimation of other users except the desired user. Therefore, our proposed detectors can be implemented blindly for both downlink and uplink of CDMA wireless communication system channels, while the method in [26] is only suitable for uplink channels of CDMA wireless communication system.

In addition, the performance gain provided by the proposed constraint depends on the property of the signature matrix $\mathbf{H}$. When the matrix $\mathbf{H}$ is of full column rank, i.e., the case of the uplink channel of CDMA wireless communication system, the proposed constraint can totally remove MAI and ISI in the statistical sense to achieve the best performance gain. When the matrix $\mathbf{H}$ is not of full rank, i.e., the case of downlink channel of CDMA wireless communication system, the proposed constraint can remove at least the influence of ISI. Table II summarizes the algorithms of the proposed receivers.

Table II

Signal processing algorithms of improved blind generalized receiver with decorrelating and blind hybrid generalized receiver

Signal frame: $\mathbf{y}(0), \mathbf{y}(1), \ldots, \mathbf{y}(P)$

STEP 1. Compute autocorrelation and cross-correlation

$\mathbf{Y}=\frac{1}{P+1} \sum_{n=0}^{P}\left[2 \mathbf{y}(n) \mathbf{x}^{H}(n)-\mathbf{y}(n) \mathbf{y}^{H}(n)+\boldsymbol{\eta}(n) \boldsymbol{\eta}^{H}(n)\right]$

$\overline{\mathbf{Y}}=\frac{1}{P+1} \sum_{n=0}^{P}\left[2 \mathbf{y}(n) \mathbf{x}^{H}(n+1)-\mathbf{y}(n) \mathbf{y}^{H}(n+1)+\boldsymbol{\eta}(n) \boldsymbol{\eta}^{H}(n+1)\right]$.

STEP 2. Compute eigendecomposition of $\mathbf{Y}$

$\mathbf{Y}=\mathbf{U}_{s} \boldsymbol{\Lambda}_{s} \mathbf{U}_{s}^{H}+\sigma^{2} \mathbf{U}_{n} \mathbf{U}_{n}^{H}$.

STEP 3. Parameter estimation

$\sigma^{2}=\frac{1}{N-L}($ Sum of themin $N-L$ eigenvalus of $\mathbf{Y}) ;$

$\mathbf{g}_{1}=\operatorname{Max}-$ eigenvect $\boldsymbol{(}\left(\overline{\mathbf{C}}_{k, u p}^{H} \mathbf{U}_{s} \mathbf{U}_{s}^{H} \overline{\mathbf{C}}_{1, u p}\right) ;$

$\mathbf{h}_{1}=\overline{\mathbf{C}}_{1, u p} \mathbf{g}_{1} ;$

$\overline{\mathbf{h}}_{1}=\overline{\mathbf{C}}_{1 k, \text { down }} \mathbf{g}_{1}$.

STEP 4. Compute $\boldsymbol{\alpha}, \overline{\boldsymbol{\alpha}}, \boldsymbol{\theta}, \overline{\boldsymbol{\theta}}, \boldsymbol{\lambda}_{1}, \overline{\boldsymbol{\lambda}}_{1}, \boldsymbol{\lambda}_{2}, \overline{\boldsymbol{\lambda}}_{2}$

$\boldsymbol{\alpha}=\mathbf{h}_{1}^{H} \mathbf{U}_{s}\left(\boldsymbol{\Lambda}_{s}-\sigma^{2} \mathbf{I}_{L}\right)^{-1} \mathbf{U}_{s}^{H} \mathbf{h}_{1} ; \overline{\boldsymbol{\alpha}}=\mathbf{h}_{1}^{H} \mathbf{U}_{s} \boldsymbol{\Lambda}_{s}^{-1} \mathbf{U}_{s}^{H} \mathbf{h}_{1} ;$ 
$\boldsymbol{\theta}=\overline{\mathbf{Y}}^{H} \mathbf{U}_{s}\left(\boldsymbol{\Lambda}_{s}-\sigma^{2} \mathbf{I}_{L}\right)^{-1} \mathbf{U}_{s}^{H} \mathbf{h}_{1} ; \overline{\boldsymbol{\theta}}=\overline{\mathbf{Y}}^{H} \mathbf{U}_{s} \boldsymbol{\Lambda}_{s}^{-1} \mathbf{U}_{s}^{H} \mathbf{h}_{1}$

$\boldsymbol{\lambda}_{2}=\left[\boldsymbol{\alpha} \overline{\mathbf{Y}}^{H} \mathbf{U}_{s}\left(\boldsymbol{\Lambda}_{s}-\sigma^{2} \mathbf{I}_{L}\right)^{-1} \mathbf{U}_{s}^{H} \overline{\mathbf{Y}}-\boldsymbol{\theta} \boldsymbol{\theta}^{H}\right]^{*}\left[\boldsymbol{\alpha} \overline{\mathbf{h}}_{1}-\boldsymbol{\theta}\right] ;$

$\overline{\boldsymbol{\lambda}}_{2}=\left[\overline{\boldsymbol{\alpha}} \overline{\mathbf{Y}}^{H} \mathbf{U}_{s} \boldsymbol{\Lambda}_{s}^{-1} \mathbf{U}_{s}^{H} \overline{\mathbf{Y}}-\overline{\boldsymbol{\theta}} \overline{\boldsymbol{\theta}}^{H}\right]^{*}\left[\overline{\boldsymbol{\alpha}}_{1}-\overline{\boldsymbol{\theta}}\right] ;$

$\lambda_{1}=\boldsymbol{\alpha}^{-1}\left[1-\boldsymbol{\theta}^{H} \lambda_{2}\right] ; \bar{\lambda}_{1}=\overline{\boldsymbol{\alpha}}^{-1}\left[1-\overline{\boldsymbol{\theta}}^{H} \bar{\lambda}_{2}\right]$.

STEP 5. Form the blind generalized receiver with decorrelating and the blind hybrid generalized receiver

$\mathbf{T}_{\text {opt }}^{d}=\mathbf{U}_{s}\left(\boldsymbol{\Lambda}_{s}-\sigma^{2} \mathbf{I}_{L}\right)^{-1} \mathbf{U}_{s}^{H}\left(\lambda_{1} \mathbf{h}_{1}+\lambda_{2} \overline{\mathbf{Y}}\right) ;$

$\mathbf{T}_{o p t}^{m}=\mathbf{U}_{s}\left(\boldsymbol{\Lambda}_{s}^{-1} \mathbf{U}_{s}^{H}\left(\bar{\lambda}_{1} \mathbf{h}_{1}+\bar{\lambda}_{2} \overline{\mathbf{Y}}\right)\right.$.

\section{Simulation Results}

In this section, simulation results are provided to demonstrate the performance of the improved blind generalized receivers and compare with the blind linear decorrelating detector and blind linear MMSE detector. We test the proposed methods in CDMA wireless communication system with spreading gain $N=31$. The spreading sequences for all users are generated by Gold codes. Both the asynchronous and synchronous cases are considered. In each case, the user 1 is assumed to be the desired user which is synchronized, and each user has $M=5$ multipath delays.

The multipath gains in each user's channel are randomly chosen and kept fixed, which have been normalized with equal power. In addition, we simulate a severe near-far case in which the power of each interfering user is $10 \mathrm{~dB}$ more than that of the desired user. In each simulation, an eigendecomposition is performed on the autocorrelation matrix of the received signals. The length of the signal frame is $P=500$. The algorithmic details of the matrix operations and parameter estimation involved in computing the various detectors can be seen from the Tables I and II.

\subsection{Performance of the proposed receivers for asynchronous CDMA wireless com- munication systems}

In the uplink of CDMA wireless communication system, the received signal at the base station is created by the asynchronous intercell and intracell users. For an asynchronous CDMA wireless communication system, we assume that the delay of each interfering user is randomly distributed within the limits of the interval $\left[0,26 T_{c}\right]$, where $T_{c}$ is the chip period. Hence, the maximum delay spread is limited within one symbol interval.

Meanwhile, it is also assumed that the signature matrix $\mathbf{H}$ in (17) is of full column rank as the existence of the delays of interfering users. We compare the performance of six receivers, i. e., the conventional blind decorrelating and MMSE detectors im- plemented by the algorithms in Table I, the group blind zero forcing and hybrid detectors investigated in [26], [40], [41] and the proposed improved blind generalized receiver with decorrelating and blind hybrid generalized receiver [42]-[44] implemented by the algorithm in Table II.

Figure 2 compares the bit-error rates $(B E R)$ of various detectors and receivers, which only require us to estimate the channel of the desired user, in an asynchronous CDMA wireless communication system with seven users. It shows that the proposed blind generalized receiver with decorrelating and blind hybrid generalized receiver provide substantial $B E R$ improvements over the conventional decorrelating and MMSE detectors. It is also observed that there exist trivial differences in performance between the proposed blind generalized receiver with decorrelating and blind hybrid generalized receiver. This is because the two receivers use the same constraint to suppress the ISI and MAI in the statistical sense. When the matrix $\mathbf{H}$ is of full rank, the proposed constraint totally removes the ISI and MAI in the statistical sense. Hence, both the proposed generalized receivers can zero force all signals from interfering users to achieve similar performance.

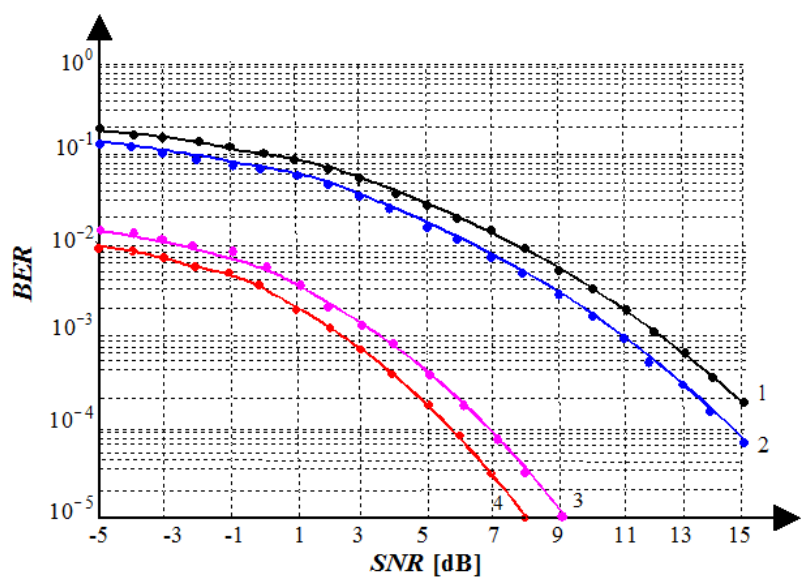

Fig. 2. BER comparison of different detectors for asynchronous CDMA $(P=500 ; K=7): 1$-conventional decorrelating detector; 2-conventional MMSE detector; 3-proposed generalized receiver with decorrelating; 4- proposed hybrid generalized receiver; solid line - theoretical calculations; $\bullet-$ simulation results.

We next compare the performance of the groupblind detectors investigated in [26] with our proposed generalized receivers. The results of the simulation are presented in Figs. 3 and 4.

In Fig. 3, ten-user asynchronous CDMA wireless communication system is considered. It is assumed that there are eight known intracell users and two unknown intercell users for the group blind detectors. It is seen that the proposed blind generalized re- 
ceiver with decorrelating has the better performance in comparison with the group-blind zero-forcing detector. The proposed blind hybrid generalized receiver has superiority over the group-blind hybrid detector by performance. It should also be noted that the proposed blind generalized receiver with decorrelating and the blind hybrid generalized receiver only need the channel estimation of the desired user, while the conventional group-blind detectors require the channel estimation of all known users.

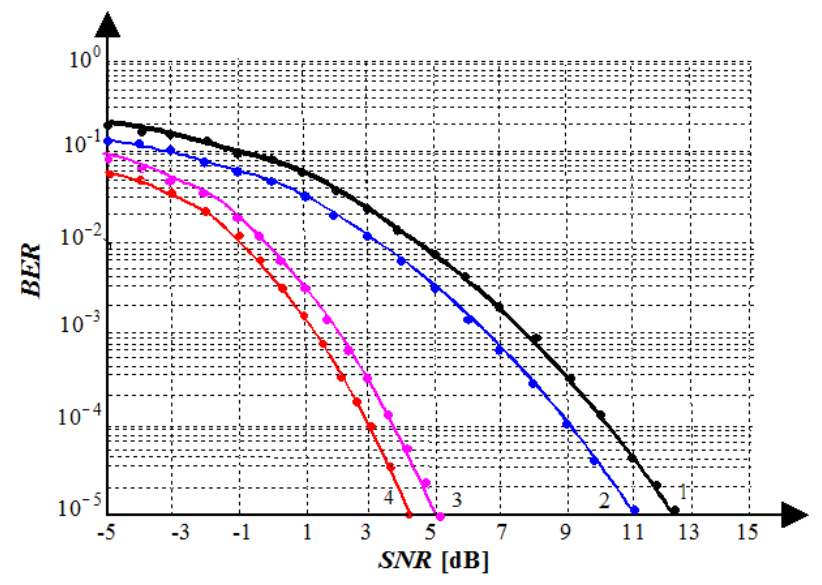

Fig. 3. BER comparison of different detectors for asynchronous CDMA $(P=500 ; K=10), 8$ known users for group detectors: 1-group-blind zero-forcing detector; 2-group-blind hybrid detector; 3-proposed generalized receiver with decorrelating; 4-proposed hybrid generalized receiver; solid line - theoretical calculations; $\bullet$ - simulation results.

Figure 4 presents a consideration of CDMA wireless communication systems with 15 users including eight intracell known users and seven intercell unknown users. Such situation may occur at the edge of the cell, where the number of the intracell users is close to the number of intercell users. It is observed that both the group-blind detectors and the proposed generalized receivers have deterioration by performance in comparison with the case presented in Fig. 3.

In this case, the performances of the proposed blind generalized receiver with decorrelating and the blind hybrid generalized receiver are marginally better than that of the group-blind zero-forcing detector and the group-blind hybrid detector, respectively. It means that the group-blind detectors are very sensitive to the number of unknown users. This is because the group-blind detectors use the channel estimation of all known users to construct the group constraint to improve the performance. Hence, the more users the detectors know the better performance gain the detectors achieve. However, the proposed generalized receivers utilize the cross-correlation of the received symbols and the channel estimation of the desired user to construct the corresponding constraint. Such constraint, which can remove both MAI and ISI in asynchronous CDMA wireless communication systems, is irrelevant to the number of unknown users.

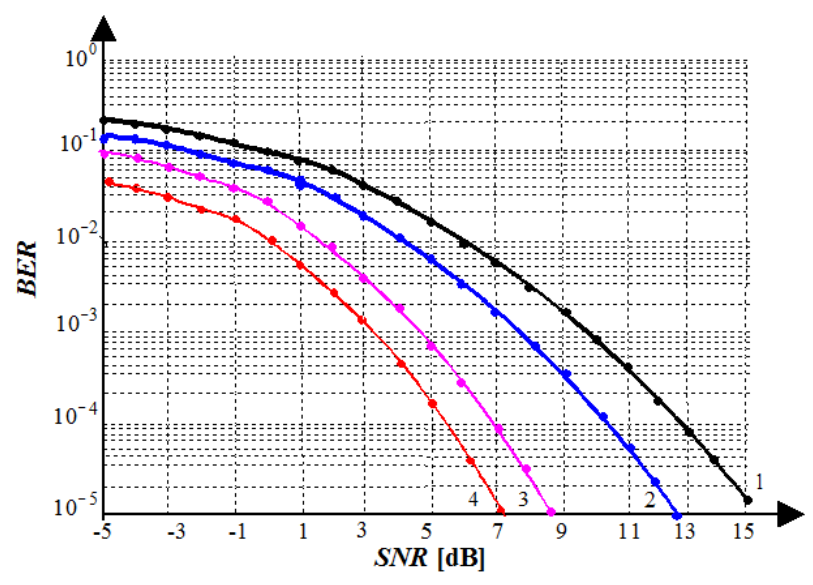

Fig. 4. BER comparison of different detectors for asynchronous CDMA $(P=500 ; K=15), 8$ known users for group detectors: 1- group-blind zero-forcing detector; 2-group-blind hybrid detector; 3-proposed generalized receiver with decorrelating; 4- proposed hybrid generalized receiver; solid line - theoretical calculations; $\bullet-$ simulation results.

In this case, the performance of the proposed blind generalized receiver with decorrelating and the blind hybrid generalized receiver is marginally better than that of the group-blind zero-forcing detector and the group-blind hybrid detector, respectively. It means that the group-blind detectors are very sensitive to the number of unknown users. This is because the group-blind detectors use the channel estimation of all known users to construct the group constraint to improve the performance. Hence, the more users the detectors know the better performance gain the detectors achieve. However, the proposed blind generalized receivers utilize the cross-correlation of the received symbols and the channel estimation of the desired user to construct the corresponding constraint. Such constraint, which can remove both MAI and ISI in asynchronous CDMA wireless communication systems, is irrelevant to the number of unknown users.

\subsection{Performance of the proposed receivers for synchronous CDMA wireless com- munication systems}

Next, we consider the performance of the proposed generalized receivers in the downlink of CDMA wireless communication system, i.e., in the synchronous CDMA wireless communication system. In the 
downlink of CDMA wireless communication system, the signal received by the desired user is made up of the synchronous intracell users and some asynchronous intercell users. In this case, the signature matrix $\mathbf{H}_{1}$, and hence the signature matrix $\mathbf{H}$, may not be of full rank when the number of synchronous intracell users surpasses the multipath length $M$.

It is noted from the above simulation results that the performance of the conventional blind MMSE detector and the proposed hybrid generalized receiver are close to the performance of the conventional blind decorrelating detector and the proposed blind generalized receiver with decorrelating, respectively. However, both the conventional blind decorrelating detector and the proposed blind generalized receiver with decorrelating require the estimate of the noise level. In addition, the group-blind detectors in [26] cannot be used for downlink channels of CDMA wireless communication system because they need to know the spreading sequences of the other intracell users.

Therefore, we only compare three detectors for the synchronous CDMA wireless communication system, i.e., the conventional MMSE detector, the proposed blind hybrid generalized receiver, and the true linear MMSE detector. The true linear MMSE detector is given by

$$
\mathbf{T}_{\text {true }}^{m}=\mathbf{Y}^{-1} \mathbf{h}_{1},
$$

where the matrix $\mathbf{Y}$ is created by the average of $10^{4}$ received samples at the high signal-to-noise ratio (SNR).

Figure 5 demonstrates comparative analysis between the performance of the above three detectors. The CDMA wireless communication system is assumed to have eight synchronous intracell users and two asynchronous intercell users. It is seen from this figure that the proposed hybrid generalized receiver still achieve the better performance in comparison with that of the conventional MMSE detector, and the performance of the conventional MMSE detector is inferior to the true linear MMSE detector one. The proposed hybrid generalized receiver is superior both the conventional MMSE detector and the true linear MMSE detector by performance in spite of the fact that because the constraint in the proposed hybrid generalized receiver can only remove the ISI in the statistical sense for synchronous CDMA wireless communication system, while it can remove both the MAI and ISI in the case of asynchronous CDMA wireless communication system in the statistical sense.

In Fig. 6 the CDMA wireless communication system has seven asynchronous intercell users and eight synchronous intracell users. In this case, the signature matrix $\mathbf{H}_{1}$ has more column rank since the number of the asynchronous users is increased. It implies that the proposed constraint can remove more MAI. It is observed as expected that the proposed hybrid generalized receiver provides more performance gain over both the conventional MMSE detector and the true linear MMSE detector by performance in comparison with Fig. 5.

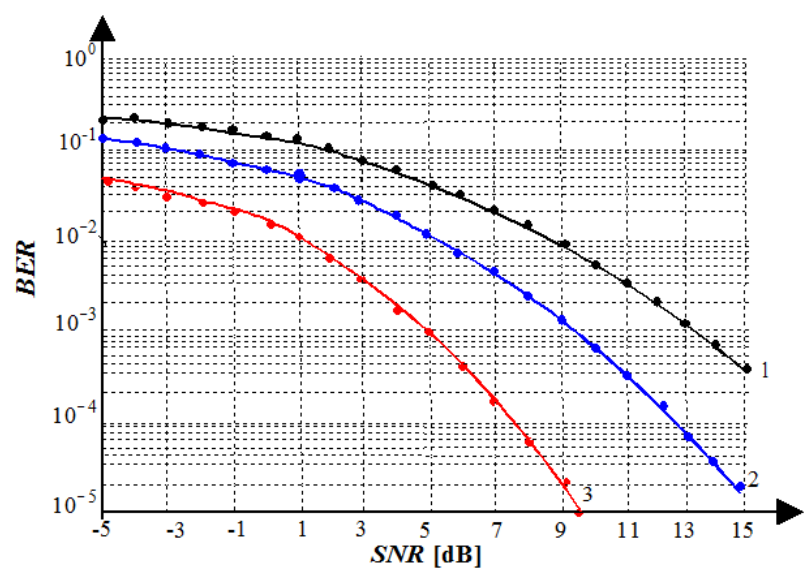

Fig. 5. BER comparison of different detectors for synchronous CDMA $(P=500 ; K=10), 8$ synchronous intracell users, 7 asynchronous intercell users: 1- conventional MMSE; 2-true MMSE; 3- proposed hybrid generalized receiver; solid line - theoretical calculations; $\bullet$ - simulation results.

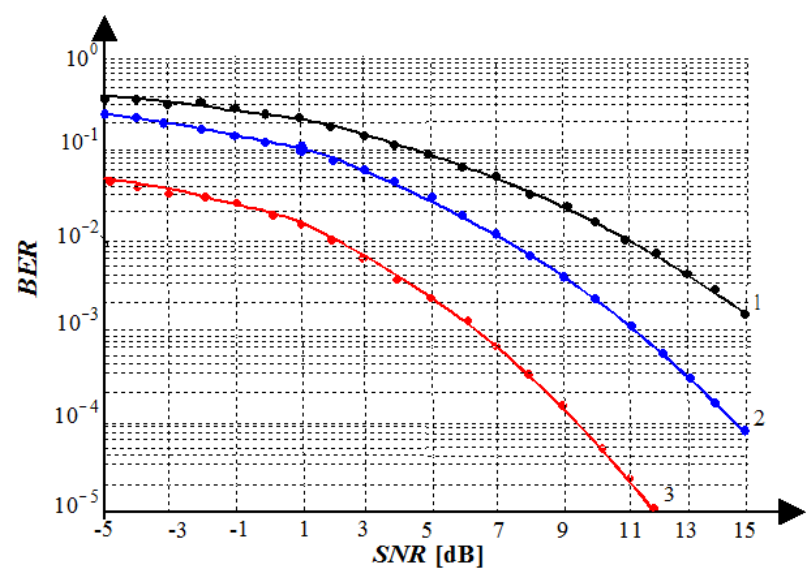

Fig. 6. BER comparison of different detectors for synchronous CDMA $(P=500 ; K=15), 8$ synchronous intracell users, 2 asynchronous intercell users: 1- conventional MMSE; 2-true MMSE; 3- proposed hybrid generalized receiver; solid line - theoretical calculations; $\bullet$ - simulation results.

Theoretically, the conventional detectors converge to the true linear MMSE detector and the proposed generalized receivers converge to the true linear generalized receiver [42]-[44] at the high $S N R$ when the signal frame length $P$ is infinite. However, for a 
finite frame length $P$, the proposed generalized receiver significantly outperforms the conventional detectors no matter in the uplink or downlink of CDMA wireless communication system. The reason for such performance improvement is that more multiuser environment information is incorporated in the derivation of the signal detection and signal processing.

\section{Conclusions}

New improved blind multiuser detection techniques are proposed for both the downlink of the synchronous CDMA wireless communication system and uplink of the asynchronous CDMA wireless communication system. The new techniques make use of an important cross-correlation matrix between the adjacent symbols as a constraint to suppress the effect of the interfering users. Two improved blind multiuser generalized receivers are developed based on different optimal criteria with the constraint constructed by the cross-correlation matrix. The proposed generalized receivers can be implemented blindly only with the channel estimation of the desired user. Simulation results show that the proposed improved blind generalized receivers provide a substantial performance gain over the conventional blind detectors.

\section{Appendix 1: Proof of Proposition 5}

Referring to the method in [26], we use Lagrange multiplier to resolve the problem in the Proposition 5. For the cost function in (61), we have

$$
\begin{gathered}
\mathbf{T}_{\text {opt }}^{d}=\underset{\mathbf{d}}{\arg \min }\left|\mathbf{d}^{H} \mathbf{H}\right|^{2} \\
+\mathscr{R}\left\{\boldsymbol{\lambda}_{1}^{*}\left(\mathbf{h}_{1}^{H} \mathbf{d}-\mathbf{1}\right)+\boldsymbol{\lambda}_{2}^{H}\left(\overline{\mathbf{Y}}^{H} \mathbf{d}-\overline{\mathbf{h}}_{1}\right)\right\} \\
=\left(\mathbf{H} \mathbf{H}^{H}\right)^{\bar{*}}\left(\boldsymbol{\lambda}_{1} \mathbf{h}_{1}+\overline{\mathbf{Y}} \boldsymbol{\lambda}_{2}\right) .
\end{gathered}
$$

Combining the constraint $\mathbf{h}_{1}^{H} \mathbf{d}=\mathbf{1}$ with (68), we obtain

$$
\mathbf{h}_{1}^{H}\left(\mathbf{H} \mathbf{H}^{H}\right)^{\bar{*}}\left(\boldsymbol{\lambda}_{1} \mathbf{h}_{1}+\overline{\mathbf{Y}} \boldsymbol{\lambda}_{2}\right)=\mathbf{1} .
$$

Hence, it is easy to obtain

$$
\boldsymbol{\lambda}_{1}=\frac{\mathbf{1}}{\mathbf{h}_{1}^{H}\left(\mathbf{H} \mathbf{H}^{H}\right)^{\overline{ }} \mathbf{h}_{1}}\left[\mathbf{1}-\mathbf{h}_{1}^{H}\left(\mathbf{H} \mathbf{H}^{H}\right)^{\bar{*}} \overline{\mathbf{Y}} \boldsymbol{\lambda}_{2}\right] .
$$

Substituting (70) into (68), we have

$$
\begin{gathered}
\mathbf{T}_{\text {opt }}^{d}=\frac{\left(\mathbf{H H}^{H}\right)^{\bar{*}}}{\mathbf{h}_{1}^{H}\left(\mathbf{H} \mathbf{H}^{H}\right)^{\bar{*}} \mathbf{h}_{1}}\left[\mathbf{h}_{1}-\mathbf{h}_{1} \mathbf{h}_{1}^{H}\left(\mathbf{H} \mathbf{H}^{H}\right)^{\bar{*}} \overline{\mathbf{Y}} \boldsymbol{\lambda}_{2}\right. \\
\left.+\mathbf{h}_{1}^{H}\left(\mathbf{H} \mathbf{H}^{H}\right)^{{ }^{*}} \mathbf{h}_{1} \overline{\mathbf{Y}} \boldsymbol{\lambda}_{2}\right] .
\end{gathered}
$$

Combining the constraint

$$
\left(\mathbf{T}_{o p t}^{d}\right)^{H} \overline{\mathbf{Y}}=\overline{\mathbf{h}}_{1}^{H},
$$

with (71) and denoting

$$
\boldsymbol{\alpha} \cong \overline{\mathbf{h}}_{1}^{H}\left(\mathbf{H} \mathbf{H}^{H}\right)^{\bar{*}} \mathbf{h}_{1},
$$

we obtain

$$
\begin{gathered}
\boldsymbol{\lambda}_{2}=\left[\boldsymbol{\alpha} \overline{\mathbf{Y}}^{H}\left(\mathbf{H} \mathbf{H}^{H}\right)^{\bar{*}} \overline{\mathbf{Y}}\right. \\
\left.-\overline{\mathbf{Y}}^{H}\left(\mathbf{H} \mathbf{H}^{H}\right)^{\overline{ }} \mathbf{h}_{1} \mathbf{h}_{1}^{H}\left(\mathbf{H} \mathbf{H}^{H}\right)^{\bar{*}} \overline{\mathbf{Y}}\right]^{\bar{*}} \\
\times\left[\boldsymbol{\alpha} \overline{\mathbf{h}}_{1}-\overline{\mathbf{Y}}^{H}\left(\mathbf{H} \mathbf{H}^{H}\right)^{\bar{*}} \mathbf{h}_{1}\right] .
\end{gathered}
$$

According to the eigendecomposition in (25), we can make the replacement

$$
\left(\mathbf{H} \mathbf{H}^{H}\right)^{\bar{*}}=\mathbf{U}_{s}\left(\boldsymbol{\Lambda}_{s}-\sigma^{2} \mathbf{I}_{L}\right)^{-1} \mathbf{U}_{s}^{H} .
$$

We then rewrite the vectors $\boldsymbol{\alpha}$ and $\lambda_{2}$ in the following form

$$
\begin{gathered}
\boldsymbol{\alpha}=\mathbf{h}_{1}^{H} \mathbf{U}_{s}\left(\boldsymbol{\Lambda}_{s}-\sigma^{2} \mathbf{I}_{L}\right)^{-1} \mathbf{U}_{s}^{H} \mathbf{h}_{1} ; \\
\boldsymbol{\lambda}_{2}=\left[\boldsymbol{\alpha} \overline{\mathbf{Y}}^{H} \mathbf{U}_{s}\left(\boldsymbol{\Lambda}_{s}-\sigma^{2} \mathbf{I}_{L}\right)^{-1} \mathbf{U}_{s}^{H} \overline{\mathbf{Y}}-\overline{\mathbf{Y}}^{H} \mathbf{U}_{s}\right. \\
\left.\times\left(\boldsymbol{\Lambda}_{s}-\sigma^{2} \mathbf{I}_{L}\right)^{-1} \mathbf{U}_{s}^{H} \mathbf{h}_{1} \mathbf{h}_{1}^{H} \mathbf{U}_{s}\left(\boldsymbol{\Lambda}_{s}-\sigma^{2} \mathbf{I}_{L}\right)^{-1} \mathbf{U}_{s}^{H} \overline{\mathbf{Y}}\right]^{\bar{*}} \\
{\left[\boldsymbol{\alpha} \overline{\mathbf{h}}_{1}-\overline{\mathbf{Y}}^{H} \mathbf{U}_{s}\left(\boldsymbol{\Lambda}_{s}-\sigma^{2} \mathbf{I}_{L}\right)^{-1} \mathbf{U}_{s}^{H} \mathbf{h}_{1}\right]} \\
=\left[\boldsymbol{\alpha} \overline{\mathbf{Y}}^{H} \mathbf{U}_{s}\left(\boldsymbol{\Lambda}_{s}-\sigma^{2} \mathbf{I}_{L}\right)^{-1} \mathbf{U}_{s}^{H} \overline{\mathbf{Y}}-\boldsymbol{\theta} \boldsymbol{\theta}^{H}\right]^{\bar{*}}\left[\boldsymbol{\alpha} \overline{\mathbf{h}}_{1}-\boldsymbol{\theta}\right],
\end{gathered}
$$

where

$$
\boldsymbol{\theta} \cong \overline{\mathbf{Y}}^{H} \mathbf{U}_{s}\left(\boldsymbol{\Lambda}_{s}-\sigma^{2} \mathbf{I}_{L}\right)^{-1} \mathbf{U}_{s}^{H} \mathbf{h}_{1} .
$$

Substituting the vectors $\boldsymbol{\alpha}$ and $\boldsymbol{\theta}$ into (70), it is easy to obtain

$$
\begin{gathered}
\boldsymbol{\lambda}_{1}=\frac{1}{\boldsymbol{\alpha}}\left[\mathbf{1}-\mathbf{h}_{1}^{H} \mathbf{U}_{s}\left(\boldsymbol{\Lambda}_{s}-\sigma^{2} \mathbf{I}_{L}\right)^{-1} \mathbf{U}_{s}^{H} \overline{\mathbf{Y}} \boldsymbol{\lambda}_{2}\right] \\
=\frac{1}{\boldsymbol{\alpha}}\left[\mathbf{1}-\boldsymbol{\theta}^{H} \boldsymbol{\lambda}_{2}\right] .
\end{gathered}
$$

Finally, the proposed generalized receiver is given by

$$
\mathbf{T}_{\text {opt }}^{d}=\mathbf{U}^{s}\left(\boldsymbol{\Lambda}_{s}-\sigma^{2} \mathbf{I}_{L}\right)^{-1} \mathbf{U}_{s}^{H}\left(\boldsymbol{\lambda}_{1} \mathbf{h}_{1}+\overline{\mathbf{Y}} \boldsymbol{\lambda}_{2}\right) .
$$

\section{Appendix 2: Proof of Proposition 6}

Similar to the Proof of Proposition 5, we use the Lagrange multiplier to resolve the problem in Proposition 6. For the cost function in (64), we have

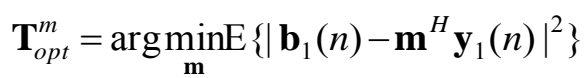

$$
\begin{aligned}
& +\mathscr{R}\left\{\boldsymbol{\lambda}_{1}^{*}\left(\mathbf{h}_{1}^{H} \mathbf{m}-\mathbf{1}\right)+\boldsymbol{\lambda}_{2}^{H}\left(\overline{\mathbf{Y}}^{H} \mathbf{m}-\overline{\mathbf{h}}_{1}\right)\right\} \\
& =\arg \min _{\mathbf{m}} \mathbf{m}^{H} \mathbf{Y} \mathbf{m}-2 \mathscr{R}\left\{\boldsymbol{\lambda}_{1}^{*}\left(\mathbf{h}_{1}^{H} \mathbf{m}\right\}\right. \\
& +\mathscr{R}\left\{\boldsymbol{\lambda}_{1}^{*}\left(\mathbf{h}_{1}^{H} \mathbf{m}-\mathbf{1}\right)+\overline{\boldsymbol{\lambda}}_{2}^{H}\left(\overline{\mathbf{Y}}^{H} \mathbf{m}-\overline{\mathbf{h}}_{1}\right)\right\} \text {. }
\end{aligned}
$$


Denoting the vector $\bar{\lambda}_{1}=\lambda_{1}-\mathbf{2}$ and substituting the vector $\bar{\lambda}_{1}$ into (81), we obtain

$$
\begin{gathered}
\mathbf{T}_{o p t}^{m}=\underset{\mathbf{m}}{\arg \min _{\mathbf{m}}} \mathbf{m}^{H} \mathbf{Y} \mathbf{m} \\
+\mathscr{R}\left\{\overline{\boldsymbol{\lambda}}_{1}^{*}\left(\mathbf{h}_{1}^{H} \mathbf{m}-\mathbf{1}\right)+\overline{\boldsymbol{\lambda}}_{2}^{H}\left(\overline{\mathbf{Y}}^{H} \mathbf{m}-\overline{\mathbf{h}}_{1}\right)\right\} \\
=\mathbf{Y}^{-\mathbf{1}}\left(\overline{\boldsymbol{\lambda}}_{1} \mathbf{h}_{1}+\overline{\mathbf{Y}}_{2}\right) .
\end{gathered}
$$

Referring to (70)-(74) and denoting

$$
\overline{\boldsymbol{\alpha}} \cong \mathbf{h}_{1}^{H} \mathbf{Y}^{-1} \mathbf{h}_{1},
$$

we have

$$
\begin{gathered}
\overline{\boldsymbol{\lambda}}_{1}=\frac{1}{\boldsymbol{\alpha}}\left[\mathbf{1}-\mathbf{h}_{1}^{H} \mathbf{Y}^{-1} \overline{\mathbf{Y}} \overline{\boldsymbol{\lambda}}_{2}\right] ; \\
\overline{\boldsymbol{\lambda}}_{2}=\left[\boldsymbol{\alpha} \overline{\mathbf{Y}}^{H} \mathbf{Y}^{-1} \overline{\mathbf{Y}}-\overline{\mathbf{Y}}^{H} \mathbf{Y}^{-1} \mathbf{h}_{1} \mathbf{h}_{1}^{H} \mathbf{Y}^{-1} \overline{\mathbf{Y}}\right]^{\bar{*}} \\
\times\left[\overline{\boldsymbol{\alpha}} \overline{\mathbf{h}}_{1}-\overline{\mathbf{Y}}^{H} \mathbf{Y}^{-1} \mathbf{h}_{1}\right] .
\end{gathered}
$$

It is seen from (40) and (49) that the vector $\mathbf{h}_{1}$ and the matrix $\overline{\mathbf{Y}} \in$ range $\left(\mathbf{U}_{s}\right)$, i.e.

$$
\mathbf{h}_{1}^{H} \mathbf{U}_{s}=\mathbf{0} \text { and } \overline{\mathbf{Y}}^{H} \mathbf{U}_{s}=\mathbf{0} .
$$

Thus, by denoting

$$
\overline{\boldsymbol{\theta}} \cong \overline{\mathbf{Y}}^{H} \mathbf{U}_{s} \boldsymbol{\Lambda}_{s}^{-1} \mathbf{U}_{s}^{H} \mathbf{h}_{1},
$$

we can rewrite the vectors $\overline{\boldsymbol{\alpha}}, \overline{\boldsymbol{\lambda}}_{1}$, and $\overline{\boldsymbol{\lambda}}_{2}$ in the following subspace form:

$$
\begin{gathered}
\overline{\boldsymbol{\alpha}}=\mathbf{h}_{1}^{H} \mathbf{U}_{s} \boldsymbol{\Lambda}_{s}^{-1} \mathbf{U}_{s}^{H} \mathbf{h}_{1} ; \\
\overline{\boldsymbol{\lambda}}_{2}=\left[\overline{\boldsymbol{\alpha}} \overline{\mathbf{Y}}^{H} \mathbf{U}_{s} \boldsymbol{\Lambda}_{s}^{-1} \mathbf{U}_{s}^{H} \overline{\mathbf{Y}}\right. \\
\left.-\overline{\mathbf{Y}}^{H} \mathbf{U}_{s} \boldsymbol{\Lambda}_{s}^{-1} \mathbf{U}_{s}^{H} \mathbf{h}_{1} \mathbf{h}_{1}^{H} \mathbf{U}_{s} \boldsymbol{\Lambda}_{s}^{-1} \mathbf{U}_{s}^{H} \overline{\mathbf{Y}}\right]^{\bar{*}} \\
\times\left[\overline{\boldsymbol{\alpha}} \overline{\mathbf{h}}_{1}-\overline{\mathbf{Y}}^{H} \mathbf{U}_{s} \boldsymbol{\Lambda}_{s}^{-1} \mathbf{U}_{s}^{H} \mathbf{h}_{1}\right] \\
=\left[\overline{\boldsymbol{\alpha}} \overline{\mathbf{Y}}^{H} \mathbf{U}_{s} \boldsymbol{\Lambda}_{s}^{-1} \mathbf{U}_{s}^{H} \overline{\mathbf{Y}}-\overline{\boldsymbol{\theta}} \overline{\boldsymbol{\theta}}^{H}\right]^{*}\left[\overline{\boldsymbol{\alpha}} \overline{\mathbf{h}}_{1}-\overline{\boldsymbol{\theta}}\right] ; \\
\overline{\boldsymbol{\lambda}}_{1}=\frac{1}{\boldsymbol{\alpha}}\left[\mathbf{1}-\mathbf{h}_{1}^{H} \mathbf{U}_{s} \boldsymbol{\Lambda}_{s}^{-1} \mathbf{U}_{s}^{H} \overline{\mathbf{Y}}_{2}\right]=\frac{1}{\boldsymbol{\alpha}}\left[\mathbf{1}-\overline{\boldsymbol{\theta}}^{H} \overline{\boldsymbol{\lambda}}_{2}\right] .
\end{gathered}
$$

Finally, the proposed detector can be given by

$$
\mathbf{T}_{\text {opt }}^{m}=\mathbf{U}_{s} \boldsymbol{\Lambda}_{s}^{-1} \mathbf{U}_{s}^{H}\left(\overline{\boldsymbol{\lambda}}_{1} \mathbf{h}_{1}+\overline{\mathbf{Y}} \overline{\boldsymbol{\lambda}}_{2}\right) .
$$

\section{References:}

[1] S. Verdu, Minimum probability of error for asynchronous multiple access communication systems, in Proceedings of IEEE Military Communications Conference. 1983, Vol.1, November 1983, pp.213-219.

[2] D.S. Chen and S. Roy, An adaptive multiuser receiver for CDMA systems. IEEE Journal on Selection Areas on Communications. 1994, Vol.12, No.6, pp.808-816.

[3] D.A. Pados and S.N. Batalama, Low-complexi- ty blind detection of DS-CDMA signals: auxillary-vector receivers. IEEE Transactions on Communications.1997, Vol. 41, No. 12, pp.15861594.

[4] U. Madhow and M.L. Honig, MMSE interference suppression for direct-sequence spread spectrum CDMA. IEEE Transactions on Communications. 1994, Vol.38, No.12, pp.3178-3188.

[5] H.V. Poor and S. Verdu, Probability error in MMSE multiuser detection. IEEE Transactions on Information Theory. 1997, Vol.43, No.5, pp. 858-871.

[6] S. Verdu, Multiuser Detection. Cambridge, U.K. Cambridge University Press, 1998, 474 pages.

[7] M. Honig, U. Madhow, and S. Verdu, Blind adaptive multiuser detection. IEEE Transactions on Information Theory. !995, Vol.41, No.7, pp.944960.

[8] X.-G. Xia, Modulated Coding for Intersymbol Interference Channels. Boca Raton, London, New York, Washington D.C., USA: CRC Press, Taylor \& Francis Group. 312 pages.

[9] X. Wang, Advanced signal processing for wireless multimedia communications. Signal Processing. 2000, Vol.3, No.1, pp.23-30.

[10] X. Wang and H.V. Poor, Wireless Communication Systems: Advanced Techniques for Signal Reception. Englewood Cliffs, NJ., USA: Prentice Hall Professional: 2004. 682 pages.

[11] X. Wang and H.V. Poor, Blind multiuser detection: a subspace approach. IEEE Transactions on Information Theory. 1998, Vol.44, No.2, pp. 677-690.

[12] G. Gelli, L. Paura, and F. Verde, Blind direct multiuser detection for uplink MC-CDMA: performance analysis and robust implementation. EURASIP Journal on Wireless Communications and Networking. 2009, Vol.1, Issue.1, pp.125-140.

[13] Y.A. Fahmy, H.-A.M. Mourad, E.K. Al-Hussainin, A generalized blind adaptive multiuser detection algorithm for multipath Rayleigh fading channel employed in a MIMO system. Journal on Communications and Networks. 2006, Vol.8, No.3, pp.290-296. 335.

[14] J.B. Schodorf and D.B. Williams, Array processing techniques for multiuser detection IEEE Transactions on Communications. 1997, Vol. 41, No.11, pp.1375-1378.

[15] A. Chkeif, K. Abed-Meraim, and G. KawasKaleh, Spatio-temporal blind adaptive multiuser detection. IEEE Transactions on Comunications. 2000, Vol.48, No.5, pp.729-732.

[16] Y. Meng, L.-M. Meng, J.-Y Hua, H. Peng, Sub- 
space-based MMSE group-blind multiuser detection algorithm. Journal on Systems Engineering and Electronics. 2009, Vol.31, Issue 7, pp.1573-1576.

[17] R. Bouziane and R.I. Killey, Blind symbol synchronization for direct detection optical OFDM using a reduced number of virtual subcarriers. Optics Express. 2015, Vol. 23, Issue 5, pp.6444 $-6454$.

[18] M. Pinchas, Inspection of the output of a convolution and deconvolution process from the leading digit point of view - Benfold's law. Journal of Signal and Information Processing. 2016, Vol.7, No.4, pp.227-251. doi:10.4236/ Jsip.2016.74020

[19] X. Ruan, C. Li, W. Yang, G. Cui, H. Zhu, Z. Zhou, Y. Dai, X. Shi, Blind sequence detection using reservoir computing. Digital Signal Processing. 2017, Vol.62, pp. 81-90.

[20] M.K. Tsatsanis and Z. Xu, Performance analysis of minimum variance CDMA receivers. IEEE Transactions on Signal Processing. 1998, Vol.49, No.1, pp.3014-3022.

[21] J. Yin, Blind adaptive multiuser detection for under-ice acoustic communications with mobile interfering users. The Journal of the Acoustical Society of America. 2017, Vol.147, Issue 7. https://doi.org/10.1121/1.4974757

[22] W. Zhou, J.K. Nelson, Blind sequential detection for sparse ISI channels. EURASIP Journal on Advances in Signal Processing. 2018, 6 (2018). https://doi.org/10.1186/s13634-017-0527-3

[23] J.A.H. Sanchez and P.E.J. Gomez, Effects of blind channel equalization using the regressive accelerator algorithm. Sistemas \& Telematica. 2018, Vo.16, No.46, pp.9-20.

[24] G. Scarano, A. Petroni, M. Biagi, R. Cusani, Blind fractionally spaced channel equalization for shallow water PPM digital communication links. Sensors. 2019, Vol. 19, No. 21: 4604; doi:10.3390/s19214604

[25] Z. Xu and M.K. Tsatsanis, Blind adaptive algorithms for minimum variance CDMA receivers. IEEE Transactions on Communications. 2001, Vol.49, No.1, pp.180-194.

[26] X.Wang and A. Host-Madsen, Group-blind multiuser detection for uplink CDMA. IEEE Journal on Selection Areas on Communications. 1999, Vol.17, No.11, pp.1971-1984.

[27] V. Tuzlukov, A new approach to signal detection theory," Digital Signal Processing. 1998, Vol.8, No.3, pp.166-184.

[28] V. Tuzlukov, Signal Detection Theory, New York: Springer-Verlag, 2001, 725 pages.
[29] V. Tuzlukov, Signal Processing Noise, Boca Raton, London, New York, Washington, D.C.: CRC Press \& Francis Group. USA, 2002, 663 pages.

[30] V. Tuzlukov, Signal and Image Processing in Navigational Systems. Boca Raton, London, New York, Washington, D.C.: CRC Press \& Francis Group. USA, 2005, 635 pages

[31] M.K. Tsatsanis and G.B. Giannakis, Optimal decorrelating receivers for DS-CDMA systems: A signal processing framework. IEEE Transactions on Signal Processing. 1996, Vol.44, pp. 3044-3055.

[32] P. Loubation and E. Moulines, On blind multiuser forward link channel estimation by the subspace method: Identifiability results. IEEE Transactions on Signal Processing. 2000, Vol. 48, No.8, pp.2366-2376.

[33] X. Wang and H.V. Poor, Blind equalization and multiuser detection in dispersive CDMA channels. IEEE Transactions on Communications. 1998, Vol.46, No.1, pp.91-103.

[34] M. Maximov, Joint correlation of fluctuative noise at outputs of frequency filters. Radio Engineering. 1956, No. 9, pp. 28-38.

[35] Y. Chernyak, Joint correlation of noise voltage at the outputs of amplifiers with no overlapping responses. Radio Physics and Electronics, 1960 No.4, pp.551-561.

[36] V. Tuzlukov, DS-CDMA downlink systems with fading channel employing the generalized detector. Digital Signal Processing, 2011. Vol. 21, No.6, pp.725-733.

[37] V. Tuzlukov, Signal processing by generalized detector in DS-CDMA wireless communication systems with frequency-selective channels. Circuits, Systems, and Signal Processing, published on-line on February 2, 2011, doi:10. 1007/s00034-011-9273-1; 2011,Vol.30, No.6, pp.1197-1230.

[38] M. Shbat and V. Tuzlukov, Primary signal detection algorithms for spectrum sensing at low SNR over fading channels in cognitive radio." Digital Signal Processing (2019). https://doi. org/10.1016/j.dsp. 2019.07.16. Digital Signal Processing. 2019. Vol.93. No.5, pp.187- 207.

[39] Shbat, M., Tuzlukov, V.P., "Evaluation of detection performance under employment of the generalized detector in radar sensor systems," Radioengineering, 2014, Vol. 23, No. 1, pp. 50-65.

[40] D. Reynolds, X. Wang, H.V. Poor, Blind adaptive space-time multiuser detection with multiple transmitter and receiver antennas. IEEE Transactions on Signal Processing. 2002, Vol. 
50, No.6, pp.1261-1276.

[41] S. Sajeed, P. Chaiwongkhot, A. Huang, et al. An approach for security evaluation and certification of a complete quantum communication system. Science Report. 2021, Vol. 11, 5110. https://doi.org/10.1038/s41598-021-84139-3

[42] M. Shbat and V. Tuzlukov, Definition of adaptive detection threshold under employment of the generalized detector in radar sensor systems. IET Signal Processing, 2014, Vol. 8, Issue 6, pp. 622-632.

[43] M. Shbat and V. Tuzlukov, SNR wall effect alleviation by generalized detector employment in cognitive radio networks. Sensors, 2015, 15 (7), pp.16105-16135;doi:10.3390/s150716105.

[44] V. Tuzlukov, Signal processing by generalized receiver in wireless communications systems over fading channels. Chapter 2 in Advances in Signal Processing. IFSA Publishing Corp. Barcelona, Spain. 2021. pp. 55-111. https://doi.org/10.1038/s41598-021-84139-3

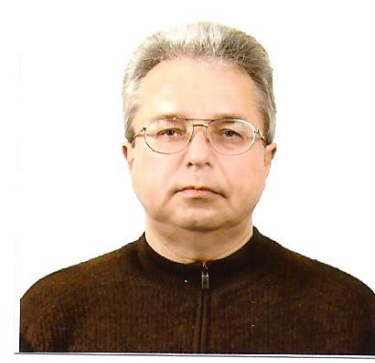

received the $\mathrm{MSc}$ and $\mathrm{PhD}$ the Belarusian State University, Minsk, Belarus in 1976 and 1990, respectively, and DSc degree in radio physics from the Kotelnikov Institute of Radioengineering and Electronics of Russian Academy of Sciences in 1995. Starting from 1995 and till 1998 Dr. Tuzlukov was a Visiting Professor at the University of San-Diego, San-Diego, California, USA. In 1998 Dr. Tuzlukov relocated to Adelaide, South Australia, where he served as a Visiting Professor at the University of Adelaide till 2000. From 2000 to 2002 he was a Visiting Professor at the University of Aizu, Aizu-Wakamatsu City, Fukushima, Japan and from 2003 to 2007 served as an Invited Professor at the Ajou University, Suwon, South Korea, within the Department of Electrical and Computer Engineering. Starting from March 2008 to February 2009 he joined as a Full Professor at the Yeungnam University, Gyeonsang, South Korea within the School of Electronic Engineering, Communication Engineering, and Computer Science. Starting from March 1, 2009 Dr. Tuzlukov served as Full Professor and Director of Signal Processing Lab at the Department of Communication and Information Technologies, School of Electronics Engineering, College of IT Engineering, Kyungpook National University, Daegu, South Korea. Currently, Dr. Tuzlukov is the Head of Department of Technical Exploitation of Aviation and Radio Engineering Equipment, Belarusian State Academy of Aviation,
Minsk, Belarus. His research emphasis is on signal processing in radar, wireless communications, wireless sensor networks, remote sensing, sonar, satellite communications, mobile communications, and other signal processing systems. He is the author over 280 journal and conference papers, seventeenth books in signal processing published by Springer-Verlag and CRC Press. Some of them are Signal Detection Theory (2001), Signal Processing Noise (2002), Signal and Image Processing in Navigational Systems (2005), Signal Processing in Radar Systems (2012), Editor of the book Communication Systems: New Research (2013), Nova Science Publishers, Inc, USA, and has also contributed Chapters "Underwater Acoustical Signal Processing" and "Satellite Communications Systems: Applications" to Electrical Engineering Handbook: $3^{\text {rd }}$ Edition, 2005, CRC Press; "Generalized Approach to Signal Processing in Wireless Communications: The Main Aspects and Some Examples" to Wireless Communications and Networks: Recent Advances, InTech, 2012; "Radar Sensor Detectors for Vehicle Safety Systems" to Electrical and Hybrid Vehicles: Advanced Systems, Automotive Technologies, and Environmental and Social Implications, Nova Science Publishers, Inc., USA, 2014; "Wireless Communications: Generalized Approach to Signal Processing" and "Radio Resource Management and Femtocell Employment in LTE Networks", to Communication Systems: New Research, Nova Science Publishers, Inc., USA, 2013; "Radar Sensor Detectors for Vehicle Safety Systems" to Autonomous Vehicles: Intelligent Transport Systems and Automotive Technologies, Publishing House, University of Pitesti, Romania, 2013; "Radar Sensor Detectors for Vehicle Safety Systems," to Autonomous Vehicles: Intelligent Transport Systems and Smart Technologies, Nova Science Publishers, Inc., New York, USA, 2014; "Signal Processing by Generalized Receiver in DS-CDMA Wireless Communication Systems," to Contemporary Issues in Wireless Communications. INTECH, CROATIA, 2014; "Detection of Spatially Distributed Signals by Generalized Receiver Using Radar Sensor Array in Wireless Communications," to Advances in Communications and Media Research. NOVA Science Publishers, Inc., New York, USA, 2015; "Signal Processing by Generalized Receiver in Wireless Communications Systems over Fading Channels" to Advances in Signal Processing. IFSA Publishing Corp. Barcelona, Spain. 2021.

He participates as the General Chair, Keynote Speaker, Plenary Lecturer, Chair of Sessions, Tutorial Instructor and organizes Special Sections at the major International Conferences and Symposia on signal processing.

Dr. Tuzlukov was highly recommended by U.S. experts of Defence Research and Engineering (DDR\& E) of the United States Department of Defence as a recognized expert in the field of humanitarian demining and minefield sensing technologies and had been awarded by Special Prize of the United States Department of Defence in 1999 Dr. Tuzlukov is distinguished as one of the leading achievers from around the world by Marquis Who's Who and his name and biography have been included in the Who's Who in the World, 2006-2013; Who's Who in World, 25 ${ }^{\text {th }}$ 
Silver Anniversary Edition, 2008, Marquis Publisher, NJ, USA; Who's Who in Science and Engineering, 20062012 and Who's Who in Science and Engineering, 10th Anniversary Edition, 2008-2009, Marquis Publisher, NJ, USA; 2009-2010 Princeton Premier Business Leaders and Professionals Honours Edition, Princeton Premier Publisher, NY, USA; 2009 Strathmore's Who's Who Edition, Strathmore's Who's Who Publisher, NY, USA; 2009 Presidental Who's Who Edition, Presidental Who's Who Publisher, NY, USA; Who's Who among Executives and Professionals, 2010 Edition, Marquis Publisher, NJ, USA; Who's Who in Asia 2012, $2^{\text {nd }}$ Edition, Marquis Publisher, NJ, USA; Top 100 Executives of 2013 Magazine, Super Network Publisher, New York, USA, 2013; 2013/2014 Edition of the Global Professional Network, Business Network Publisher, New York, USA, 2013;
2013/2014 Edition of the Who's Who Network Online, Business Network Publisher, New York, USA, 2014; Online Professional Gateway, 2014 Edition, Business Network Publisher, New York, USA, 2014; 2014 Worldwide Who's Who", Marquis Publisher, NJ, USA; 2015 Strathmore Professional Biographies, Strathmore's Who's Who Publisher, NY, USA; Who's Who in World, 2015, Marquis Publisher, NJ, USA; 2015-2016 Membership in Exclusive Top 100 network of professionals in the world, NY, USA, 2015; 2015 Who's Who of Executives and Professionals Honors Edition, Marquis Publisher, NJ, USA; Worldwide Who's Who - Top 100 Business Networking, San Diego, CA, USA, 2015.

Phone: +375173453283

Email: slava.tuzlukov@mail.ru

Creative Commons Attribution License 4.0 (Attribution 4.0 International, CC BY 4.0)

This article is published under the terms of the Creative Commons Attribution License 4.0 https://creativecommons.org/licenses/by/4.0/deed.en_US 